NOTICE: this is the author's version of a work that was submitted for publication in Electric Power Systems Research. Changes resulting from the reviewing, publishing process, such as peer review, editing, corrections, structural formatting, and other quality control mechanisms may not be reflected in this document. Changes may have been made to this work since it was submitted for revision and publication. A definitive version was subsequently accepted and published in Electric Power Systems Research, DOI: 10.1016/j.epsr.2013.08.006 


\title{
Optimization Models for an EV Aggregator Selling Secondary Reserve in the Electricity Market
}

\author{
R.J. Bessa* and M.A. Matos \\ INESC TEC - INESC Technology and Science (formerly INESC Porto) and FEUP - Faculty of Engineering, University of Porto, \\ Portugal
}

\begin{abstract}
Power system regulators and operators are creating conditions for encouraging the participation of the demand-side into reserve markets. The electric vehicle (EV), when aggregated by a market agent, holds sufficient flexibility for offering reserve bids. Nevertheless, due to the stochastic nature of the drivers' behavior and market variables, forecasting and optimization algorithms are necessary for supporting an EV aggregator participating in the electricity market. This paper describes a new day-ahead optimization model between energy and secondary reserve bids and an operational management algorithm that coordinates EV charging in order to minimize differences between contracted and realized values. The use of forecasts for EV and market prices is included, as well as a market settlement scheme that includes a penalty term for reserve shortage. The optimization framework is tested in a test case constructed with synthetic time series for EV and market data from the Iberian market.
\end{abstract}

Keywords: Electric vehicle, aggregator, optimization, electricity market, secondary reserve, regulation reserve.

\section{Nomenclature}

$\mu$ : ratio between upward and downward secondary reserve;

$\Psi:$ costs associated to deviations between actual charging and accepted bids;

$\varphi$ : convex loss function;

$\Phi$ : costs associated to reserve shortage;

$\alpha$ : penalization coefficient for secondary reserve capacity shortage;

$\gamma$ : penalization coefficient for reserve not supplied (electrical energy);

$\Delta t$ : time step (length of the time interval) of time interval $t$;

$E_{t}$ : optimized electrical energy for time interval $t$;

$E_{t, j}$ : optimized electrical energy for charging the $\mathrm{j}^{\text {th }} \mathrm{EV}$ in time interval $t$;

*Correspondence to: Ricardo Bessa, INESC Porto, Campus da FEUP, Rua Dr. Roberto Frias, 378, 4200 - 465 Porto Portugal. Telf: +35122209 4208. Fax: +35122209 4050. E-mail: rbessa@inescporto.pt 
$E_{t, j}^{*}$ : electrical energy consumed by the $\mathrm{j}^{\text {th }} \mathrm{EV}$ in time interval $t$;

$H$ : set of time intervals from the optimization horizon;

$\hat{H}_{j}^{\text {plug }}$ : forecasted availability (or plugged-in) period of the $\mathrm{j}^{\text {th }} \mathrm{EV}$;

$H_{j}^{\text {plug }}$ : availability (or plugged-in) period of the $\mathrm{j}^{\text {th }} \mathrm{EV}$;

$\lambda_{t}^{u p}:$ number of equivalent minutes of dispatched upward reserve in interval $t$;

$\lambda_{t}^{\text {down }}$ : number of equivalent minutes of dispatched downward reserve in interval $t$;

$M_{t}$ : total number of EV plugged-in at time interval $t$;

$\pi_{t}^{-}$: negative imbalance unit cost of time interval $t$;

$41 \pi_{t}^{+}$: positive imbalance unit cost of time interval $t$;

$P_{j}^{\max }$ : maximum charging power of the $\mathrm{j}^{\text {th }} \mathrm{EV}$;

$43 \bar{P}_{t_{0}}^{\max }$ : maximum, constant and feasible charging power of the EV fleet in time interval $t_{0}$.

$44 \bar{P}_{t_{0}}^{\min }$ : minimum, constant and feasible charging power of the EV fleet in time interval $t_{0}$.

$P_{t, j}^{\text {down }}:$ downward secondary reserve power of the $\mathrm{j}^{\text {th }} \mathrm{EV}$ for time interval $t$;

$P_{t, j}^{u p}:$ upward secondary reserve power of the $\mathrm{j}^{\text {th }} \mathrm{EV}$ for time interval $t$;

$47 \quad P_{t_{0}}^{\prime}$ : operating point (or actual preferred operating point);

$49 \quad P_{t_{0}}^{\prime \text { up }}$ : available upward secondary reserve power;

$50 \quad \bar{P}_{t_{0}}^{\text {down }}$ : downward secondary reserve power that can be sustained during interval $t$

$51 \quad \bar{P}_{t_{0}}^{u p}$ : upward secondary reserve power that can be sustained during interval $t$

$52 \bar{P}_{t_{0}}^{\text {upper }}$ : upper power limit that guarantees full availability of downward reserve power in time interval $t_{0}$;

$53 \bar{P}_{t_{0}}^{\text {lower }}$ : lower power limit that guarantees full availability of upward reserve power in time interval $t_{0}$;

$54 \quad p_{t}^{\text {surplus }}$ : price for positive imbalances of time interval $t$;

$55 p_{t}^{\text {shortage }}:$ price for negative imbalances of time interval $t$;

$56 \hat{p}_{t}$ : day-ahead energy price forecast for time interval $t$;

$57 \hat{p}_{t}^{c a p}$ : forecasted capacity price of secondary reserve;

$58 \hat{p}_{t}^{\text {down }}$ : forecasted price for dispatched downward reserve; 
$\hat{p}_{t}^{u p}$ : forecasted price for dispatched upward reserve;

$p_{t}$ : day-ahead energy price for time interval $t$;

$61 \hat{R}_{j}$ : forecasted charging requirement of the $\mathrm{j}^{\text {th }} \mathrm{EV}$;

$R N S_{t}^{u p}$ : upward reserve not supplied in time interval $t$

$T$ : time interval of the last plugged-in EV to depart;

$t_{\text {final }}$ : last time interval of the availability period; $v_{k}$ : slack variable;

The participation of loads in ancillary services markets has gained relevance in the recent years [1], in particular with the deployment of the smart-grid concept with bidirectional communication [2]. The electric vehicle (EV), when aggregated by a market agent, is a suitable candidate for selling reserve services in the electricity market [3].

Secondary (or regulation) reserve consists in loads and generators under direct real-time control of the system operator (SO), via automatic generation control (AGC), for increasing or decreasing generation/consumption. The response time is very fast (e.g., less than 30 seconds) and is used to bring back the frequency and the interchange programs to their nominal values (i.e., reduce the area control error - ACE)

The current market rules do not allow the participation of small loads and generators (e.g., the minimum bid is generally around megawatts), and even if small bids are allowed, the AGC would need to send control signals to each EV supplying secondary reserve.

The solution proposed by several authors is an EV aggregator acting as an intermediary between EV drivers, the electricity market and the SO [4][5]. Almeida [6] describes a control scheme for integrating aggregated EV in the AGC operation of interconnected systems. In this framework, the AGC sends setpoints to aggregators that, afterwards, distribute individual set-points among the plugged-in EV. This reduces significantly the communication burden and increases its reliability. 
EV plugged-in in slow charging points and sells secondary reserve power in the electricity market.

The vehicle-to-grid (V2G) mode was not considered in this paper. Instead, the reserve is supplied by establishing a preferred operating point (POP) [7]. The POP consists in the EV consumption level that can be increased (downward reserve) or decreased (upward reserve) limited by zero and by the maximum charging power. For instance, an EV charging at $2 \mathrm{~kW}$ could provide $2 \mathrm{~kW}$ of upward regulation until it reaches "zero load" and $1 \mathrm{~kW}$ of downward regulation if the maximum charging power is $3 \mathrm{~kW}$. Compared to $\mathrm{V} 2 \mathrm{G}$, this solution does not require additional investment in equipment, and it reduces the costs with battery wear and losses in the charger [7].

Different algorithms for supporting the participation of EV in the reserve market were proposed in the literature. Sortomme and El-Sharkawi [8] propose three heuristic strategies and equivalent optimal analogues to define the POP and regulation reserve bids of an EV aggregator. Han et al. [9] describe a dynamic programming based algorithm to calculate regulation power bids from EV. Rotering and Ilic [10] describe two dynamic programming optimization algorithms for an optimal controller installed in an EV. One algorithm optimizes the charging rates and periods for minimizing the cost, and the other maximizes the profit from selling regulation power. Wu et al. [11] discuss pricing schemes to induce the participation of EV in frequency regulation services.

All the aforementioned algorithms assume that perfect forecasts are available for all the variables. In fact, when designing bidding optimization models, it is necessary to consider the need to forecast these variables and the occurrence of forecast errors. Pantos [12] presents a stochastic optimization algorithm for the participation in the electricity market (energy and regulation reserve), which includes uncertainties related to the market price and driver's behavior. Han et al. [13] propose a probabilistic model for modeling the achievable power capacity of an EV aggregator when providing regulation reserve. Bessa et al. [14] described an optimization model for energy and secondary reserve bids. A naïve forecasting approach was used for producing forecasts for aggregated values of the EV variables. Bessa and Matos [15] compared two alternative approaches to optimize the participation of an EV aggregator in the dayahead energy market (reserve was not considered). The two algorithms use, as input, forecasts for the EV variables produced by statistical models. The same authors present in [16] a day-ahead optimization model and operational management algorithms for day-ahead and hour-ahead manual (or balancing) reserve bids.

Compared to Pantos [12] and Han et al. [13], the optimization approach proposed in this present paper 
characterizes the EV individually, which as shown in [15], provides a more accurate representation and coordinates the EV individual charging for mitigating forecast errors. Furthermore, the formulation of the optimization models proposed in this present paper contemplates the specific characteristics of secondary reserve. For instance, the models that will be described in section 3 are formulated to be robust to the variability (in size and direction) of the net electrical energy from the secondary reserve dispatch. The influence of forecast errors is also studied, in particular its impact on reserve shortage situations, and a market settlement scheme with a penalty term for reserve shortage situations is also proposed. Finally, an operational management algorithm is also described, which is essential to coordinate the EV charging during the operating hour to comply with the market commitments, while in [12] this was identified as future work.

Compared to the approach described by Bessa et al. [14], the present paper makes several innovations: the formulation of the optimization problem includes the possibility of offering a reserve band in both upward and downward directions; it disregards the need to forecast the reserve direction and participation factor; the optimization uses forecasts for each EV; an operational management algorithm is proposed for coordinating EV charging and for minimizing the difference between contracted and realized values of energy and reserve. Compared to the approach described by Bessa and Matos [16] for the manual reserve, the day-ahead and operational management problems described in this paper are different, since they were developed taking into account the characteristics of secondary reserve. For example, the proposed dayahead optimization model does not derive the reserve bids based on the forecasted reserve direction (that was found to be almost random), but it offers a reserve band in both directions and the operational management algorithm is based on a strategy that redefines the EV fleet's operating point in order to maximize the available secondary reserve.

The remaining of the paper is organized as follows: section 2 describes the problem and the specific characteristics of secondary reserve; section 3 formulates the day-ahead optimization problem; section 4 describes the operational management algorithm and how the aggregator redefines the EV fleet's operating point; section 5 proposes two new market settlement schemes; the test case results are presented and discussed in section 6; section 7 presents the overall conclusions.

\section{Problem Description}

\subsection{Electricity Market Framework}

The EV aggregator participates in the day-ahead electrical energy market with bids to purchase energy, 
which are paid at a single marginal price.

In addition to this market session, a day-ahead session for secondary reserve capacity is also considered. Two examples of market sessions for this reserve type are the secondary reserve market in the Iberian electricity market (MIBEL) [17] and the regulation reserve market in CAISO (California ISO) [18].

This reserve is generally contracted in a day-ahead basis (e.g. Portugal, Spain, Italy and the Alberta region), and even in markets with hour-ahead sessions, a major fraction of the reserve is contracted dayahead (see the case of CAISO [18]). There are two possible market-clearing schemes: a sequential market (typically European markets) where the energy market takes place first, followed by a market for secondary reserve; a market where energy and reserve requirements are jointly cleared (typically U.S. markets). The approach described in this paper makes no distinction between these two schemes, but a sequential market-clearing is assumed in this paper since the Iberian market is used as test case in section 6.

The aggregator presents a bid with a reserve band (in MW) that is divided into upward and downward directions, and the reserve is remunerated with two prices: available capacity price (in $€ / \mathrm{MW}$ ) that results from the capacity allocation of the secondary reserve market; dispatched capacity price (in $€ / \mathrm{MWh}$ ) that may result from the balancing market.

The aggregator is a price-taker, which means that the bids made by the aggregator do not affect the market-clearing price of energy and reserve. The price-taker assumption is valid when there is sufficient competition in the market and a single market agent does not have a large quota of the market (i.e., market power). Nevertheless, if the size of the aggregator's bid becomes significant, even if it remains a price-taker, it will shift the merit order curve and change the market-clearing price. In this case, it is not possible to decouple the price forecast from the buying/selling bids computed with the optimization problem.

In general, the electricity markets have hourly or half-hourly time steps. For the secondary reserve market, the power in the reserve bid is assumed to be constant during the market interval. An EV aggregator may not be able to offer constant power during a complete hour because several EV can depart and arrive during that interval. For instance, the aggregator can have $1000 \mathrm{EV}$ plugged-in during a halfhour and $800 \mathrm{EV}$ during the second half-hour. If all EV are charging at $2 \mathrm{~kW}$ (but with a maximum charging power of $3 \mathrm{~kW}$ ), the aggregator can offer $1 \mathrm{MW}$ of downward reserve in the first half-hour and 
$0.8 \mathrm{MW}$ in the second. However, in an hourly time interval, the average power would be $0.9 \mathrm{MW}$, which can only be attained during the first half-hour.

Therefore, in this paper a change in the current market rules is assumed to promote the participation of $\mathrm{EV}$ in secondary reserve. The market time interval remains one hour, which means that from the marketclearing it results an hourly price, but the secondary reserve bid submitted by the EV aggregator is decomposed in sub-hourly intervals of predefined length $\Delta t$ and with constant power. In the aforementioned example, assuming $\Delta t$ equal to 30 minutes, the downward reserve bid would be: $1 \mathrm{MW}$ for the first half-hour and 0.8 MW for the second. The time length $\Delta t$ is a predefined value and it should be defined in accordance to the average trip duration time. Note that most of the electricity markets created complex bids to accommodate specific characteristics of conventional generation units (e.g., minimum run times). Thus, this can be seen as an additional complex bid designed for EV aggregators (and also for other types of flexible loads). This change demands a new market-clearing algorithm that takes into account complex bids from the EV aggregator.

\subsection{Characteristics of the Secondary Reserve}

In the absence of perturbations, the events handled by secondary reserve are usually minute-to-minute random fluctuations inside the operating period, but in some cases, this reserve can also be used to handle large deviations between load and generation (e.g. unplanned outage or loss of synchronism from a generator). Despite being contracted on an hourly basis, the secondary reserve is mobilized for short periods-of-time (e.g., 5 minutes). Secondary reserve must only be used to correct the ACE and not for other purposes, such as to minimize unintentional energy imbalances [19].

This contrasts with manual (or balancing) reserve that is frequently used for periods of more than one hour to solve energy imbalances, such as forecast errors from renewable energy. According to Hirst [20], manual reserve (called load-following by the author) differs from secondary (called regulation by the author) in two important aspects: (a) it is used over long periods of time compared to secondary reserve; (b) the changes in reserve direction are frequently predictable and have similar daily patterns [16].

This reserve has specific characteristics that must be considered when developing optimization models for an EV aggregator.

The first characteristic is that, despite being contracted on an hourly basis, secondary reserve is normally not dispatched in the same direction during the complete hour. In an hourly period, the reserve can be dispatched in one direction during a period below one hour (e.g., upward reserve during 40 
minutes), while in other cases, it can be dispatched in both directions (e.g., 10 minutes of upward and 50 minutes of downward reserve).

Figure 1 depicts the histograms for the number of equivalent minutes of dispatched secondary upward reserve of a hydro and a thermal power plant in Portugal. The number of equivalent minutes corresponds to the ratio between the dispatched reserve power (energy in $\mathrm{MWh}$ ) and its available reserve power (power in MW).

Figure 1: Histograms for the number of equivalent minutes of the upward secondary reserve of a hydro

(Alqueva) and thermal (Lares) power plants in Portugal for the year 2011.

The two histograms show a wide variation of the number of equivalent minutes. This means that, when making a reserve bid, the aggregator does not know, with certainty, the reserve dispatch duration. For example, for a downward reserve bid of $1 \mathrm{MW}$, a value of 20 minutes in the histogram corresponds to dispatching this reserve power only during 20 minutes and no dispatch in the remaining 40 minutes and, in this case, the EV fleet only charges $0.33 \mathrm{MWh}$ of electrical energy (instead of the expected $1 \mathrm{MWh}$ ). In contrast to generation units, this creates a problem for EV since their charging requirements must be satisfied and the aggregator does not know beforehand, with certainty, the quantity of electrical energy charged as downward reserve. The same is valid for upward reserve.

The number of equivalent minutes of dispatched secondary reserve is generally low. For instance, the annual average value of the hydropower plant is 22 minutes for upward and 24 minutes for downward secondary reserve.

A second characteristic, and in contrast to the assumption made in literature about the EV aggregator participation in the secondary reserve market (see for instance reference [9]), is that the net electrical energy from reserve provision in each hour is different from zero. Figure 2a depicts the histogram of the total net energy of secondary reserve in Portugal, during the year 2011. As shown in the histogram, the net energy is frequently different from zero. An asymmetrical regulation signal adds uncertainty to the battery state of charge after each hour.

Figure 2: (a) Histogram of the net electrical energy of secondary reserve in Portugal for the year 2011 (negative value is upward reserve, positive is downward reserve); (b) Autocorrelation function (ACF) of the net electrical energy of secondary reserve in Portugal for the year 2011.

A third characteristic, and linked to the second one, is that it is challenging to produce forecasts with acceptable accuracy for this net energy. Figure $\mathbf{2 b}$ depicts the autocorrelation plot of the total net energy of secondary reserve in Portugal, during the year 2011. This plot shows an autocorrelation below 0.25 for 
all time lags, and the value for $t-1$ is only around 0.25 . This low value of serial dependency suggests that there is a low amount of information in the past values of the time series, which makes it challenging to produce forecasts with acceptable accuracy. This is consistent with the expected random nature of the secondary reserve dispatch.

To conclude, the analyses conducted in this section showed the following:

- the duration period of the dispatched reserve is variable, and in general, lower than one hour;

- the net energy from the reserve dispatch is frequently different from zero, and it is difficult to forecast its value with acceptable accuracy.

Therefore, the formulation of the day-ahead optimization problem, which will be presented in section 3, should include constraints that allow a degree of flexibility in handling situations where the available reserve in the previous intervals was not dispatched in one direction (on the contrary to what was planned by the aggregator) or was dispatched only for a limited period of time in one direction.

\subsection{Participation in the Electricity Market}

Figure 3 depicts the sequence of tasks for the participation in the day-ahead energy and secondary reserve markets. The gate closure and period for submitting bids are the ones from the Iberian electricity market.

Figure 3: Sequence of tasks for the participation in the day-ahead energy and secondary reserve markets.

In the first phase, the aggregator, at day $\mathrm{D}$, forecasts the EV charging requirement and availability, the energy, and reserve prices (described in section 3.1). This forecasted information is the input, in a second phase, of a day-ahead optimization model (for next day D+1) that computes the bids for the energy and secondary reserve markets (described in section 3.2).

During the operating day (day $\mathrm{D}+1$ ), before the beginning of each time interval $t_{0}$ (with length $\Delta t$ ), the aggregator redefines the EV fleet operating point, computes the available upward and downward reserve power, and communicates this information to the SO (described in section 4.1). The aggregator dispatches the EV for meeting the fleet's operating point for each time interval $\left(t_{0}, t_{0+1}, \ldots\right)$ and places the plugged-in $\mathrm{EV}$ on standby to supply upward and downward reserve in response to an AGC request. An operational management algorithm is used to coordinate the EV charging (described in section 4.2). A penalty term is applied for cases with reserve power shortage.

\section{Day-ahead Energy and Reserve Optimization}

Section 2.2 discussed the characteristics of secondary reserve and concluded that it is not possible to 
produce forecasts with acceptable quality for the hourly AGC regulation signal. Thus, the formulation of the day-ahead optimization problem described in this section disregards this information, and the goal is to obtain robust solutions that assure an acceptable reliability of the secondary reserve provision as well as an attractive income to the aggregator and the EV in its portfolio.

The algorithm uses, as input, forecasts for several variables that are briefly described in section 3.1.

\subsection{Input Variables and Forecasts}

The EV load is modeled with two variables: availability period and charging requirement. The EV availability is the time-period when the EV is plugged-in for charging. It is a binary variable indicating whether or not the EV is plugged-in for charging in each time interval with length $\Delta t$.

The charging requirement of the EV is the total energy needed to get from the initial state-of-charge (SOC) (i.e., when the EV arrives for charging) to the target SOC defined by the EV driver for the next trip, including the losses from the charger. A charging requirement value is always associated to an availability period. For example, an EV with battery size of $24 \mathrm{kWh}$ parking with a $50 \%$ SOC (12 kWh) and with target SOC of $100 \%$, needs $12 \mathrm{kWh}$ to reach full battery plus $1.33 \mathrm{kWh}$ of charger losses. Thus, the charging requirement is $13.33 \mathrm{kWh}$.

These variables are obtained from the advanced metering infrastructure installed in households. In this framework, it is assumed that the EV driver, when plugged-in for charging, communicates the target SOC and expected departure hour to the aggregator. If this information is not communicated, the aggregator will assume a target SOC of $100 \%$ by default.

The availability period is a binary time series forecasted with a generalized linear model (GLM) [21] with the response variable following a binomial distribution. After forecasting the availability period, the corresponding charging requirement is forecasted with non-parametric bootstrapping. A complete description of the forecasting algorithm can be found in [15].

The day-ahead energy price is forecasted with an additive model (using cubic splines) and using the following variables as explanatory variables: lagged variables of the price (i.e., $t-1, t-2, t-3$ ), forecasted wind power penetration, periodic function for the hour of the day and day of the week.

The secondary reserve has two prices: price for available reserve capacity and price for dispatched reserve. The price for available reserve capacity is forecasted with an ARIMA model selected using the function auto.arima $\mathrm{R}$ package forecast [22]. The price for dispatched reserve is an irregular time series forecasted with the Holt-Winters model with trigonometric functions [23]. 
The decision variables of the optimization problem are: optimized energy $\left(E_{t, j}\right)$ for charging the $\mathrm{j}^{\text {th }} \mathrm{EV}$ in time interval $t$ (i.e., the preferred operation point - POP), the upward and downward secondary reserve power $\left(P_{t, j}^{\text {down }}\right.$ and $\left.P_{t, j}^{u p}\right)$ of the $\mathrm{j}^{\text {th }} \mathrm{EV}$ for time interval $t$. The energy and reserve bids are the sum of the individual values of each EV (i.e., the decision variables associated to each $\mathrm{EV}-E_{t, j}, P_{t, j}^{\text {down }}$ and $P_{t, j}^{u p}$ ).

The optimization problem is formulated assuming that there is a single reserve capacity price. In markets with separated sessions for upward and downward secondary reserve, the modification would be a different capacity price for each direction.

The objective function is the minimization of the total cost, and it has the following components: (a) cost of purchasing energy; (b) income from reducing the consumption (dispatched upward reserve); (c) cost from charging EV as downward reserve; (d) income from having available secondary reserve power. It can be written as:

$$
\min \sum_{t \in H}\left(\begin{array}{c}
\hat{p}_{t} \cdot \sum_{j=1}^{M_{t}}\left(E_{t, j}\right)-\hat{p}_{t}^{u p} \cdot \sum_{j=1}^{M_{t}}\left(P_{t, j}^{u p} \cdot \Delta t\right)+ \\
\hat{p}_{t}^{\text {down }} \cdot \sum_{j=1}^{M_{t}}\left(P_{t, j}^{\text {down }} \cdot \Delta t\right)-\hat{p}_{t}^{\text {cap }} \cdot \sum_{j=1}^{M_{t}}\left(P_{t, j}^{\text {up }}+P_{t, j}^{\text {down }}\right)
\end{array}\right)
$$

where $\hat{p}_{t}$ is the forecasted energy price, $\hat{p}_{t}^{u p}$ is the forecasted price for dispatched upward reserve, $\hat{p}_{t}^{\text {down }}$ is the forecasted price for dispatched downward reserve, $\hat{p}_{t}^{\text {cap }}$ is the forecasted price for available reserve capacity, $M_{t}$ is the number of EV plugged-in at time interval $t, \Delta t$ is the length of time interval $t, H$ is the set of time intervals of the optimization period (e.g., for one day with $\Delta t=0.5 \mathrm{hr}, H$ ranges between 1 and 48).

The constraints of the optimization problem are described in the following paragraphs.

The method for computing the reserve band is as follows: first, the charging requirements are satisfied considering the purchased energy and the upward reserve band, and then, the downward capacity is the remaining capacity (below the maximum charging power, $P_{t}^{\max }$ ) in each time interval $t$.

The first point leads to the following constraint:

$$
\sum_{t \in \hat{H}_{j}^{\text {plug }}}\left(E_{t, j}-P_{t, j}^{u p} \cdot \Delta t\right)=\hat{R}_{j}, \forall j \in\left\{1, \cdots, M_{t}\right\}
$$

where $\hat{R}_{j, i}$ is the forecasted charging requirement of the $\mathrm{j}^{\text {th }} \mathrm{EV}$, and $\hat{H}_{j}^{\text {plug }}$ is the forecasted availability period of the $j^{\text {th }} \mathrm{EV}$.

The second point leads to the following constraint for downward reserve: 


$$
E_{t, j} / \Delta t+P_{t, j}^{\text {down }} \leq P_{j}^{\max }, \forall j \in\left\{1, \cdots, M_{t}\right\}, \forall t \in H
$$

The upward reserve band is limited by the energy bid in each time interval:

$$
P_{t, j}^{u p} \leq\left(E_{t, j} / \Delta t\right), \forall j \in\left\{1, \cdots, M_{t}\right\}, \forall t \in H
$$

and its total is limited by the charging requirement in each availability period:

$$
\sum_{t \in \hat{H}_{j}^{\text {plug }}}\left(P_{t, j}^{u p} \cdot \Delta t\right) \leq \hat{R}_{j}, \forall j \in\left\{1, \cdots, M_{t}\right\}
$$

Constraint (5) is included to avoid the aggregator from offering a total upward reserve greater than the total energy that the EV fleet can consume (i.e., the charging requirement). For example, without this constraint, an EV parked for 10 hourly intervals with a forecasted charging requirement of $1.5 \mathrm{kWh}$ could offer upward reserve in 9 intervals. This would give $\sum_{t \in \hat{H}^{\text {phs }}}\left(E_{t, j}\right)=10 \cdot 1.5=15 \mathrm{kWh}$ and $\sum_{t \in \hat{H}_{j}^{\text {phus }}}\left(P_{t, j}^{u p} \cdot \Delta t\right)=9 \cdot 1.5=13.5 \mathrm{kWh}$ for meeting the charging requirement. If in one of these intervals upward reserve is not dispatched, this strategy would harm significantly the reliability of upward reserve and increase the penalty costs for reserve shortage (topic that will be discussed in section 5); the inclusion of constraint (5) limits $\sum_{t \in \hat{H}_{j}^{\text {phs }}}\left(P_{t, j}^{u p} \cdot \Delta t\right) \leq 1.5 \mathrm{kWh}$.

The total downward reserve is also constrained by the charging requirement:

$$
\sum_{t \in \hat{H}_{j}^{\text {plug }}}\left(P_{t, j}^{\text {down }} \cdot \Delta t\right) \leq \hat{R}_{j}, \forall j \in\left\{1, \cdots, M_{t}\right\}
$$

With the constraint (7), the aggregator can only offer upward reserve in a specific interval if the EV is able to offer an energy bid $\left(E_{k, j}\right)$ with the corresponding quantity both in the same and subsequent time intervals. This increases the robustness of the bidding optimization since it forces the EV to be capable of consuming the quantity that is offered as upward reserve. Otherwise, considerable penalization (topic discussed in section 5) could be incurred if upward reserve cannot be supplied. This constraint consists in postponing EV charging by offering upward reserve:

$$
\sum_{k=t}^{k=t_{\text {final }}}\left(P_{k, j}^{u p} \cdot \Delta t\right) \leq \sum_{k=t}^{k=t_{\text {final }}}\left(E_{k, j}\right) / 2, \quad \forall j \in\left\{1, \cdots, M_{t}\right\}, \forall t \in H
$$

where $t_{\text {final }}$ is the last time interval of the forecasted availability period, i.e. $t_{\text {final }} \in \hat{H}_{j}^{\text {plug }}$.

In (7), the total consumption reduction between $t$ and $t_{\text {final }}$ must be below or equal to half of the energy bid in the same period. For example, if the aggregator in time interval $t=1$ offers an energy and upward reserve bid of $1.5 \mathrm{~kW}$, it must present an additional energy bid of $1.5 \mathrm{kWh}$ in any interval $t>1$ of the availability period, otherwise the constraint is violated. 
In order to illustrate this constraint, Table 1 presents two candidate solutions for offering upward reserve with an EV plugged-in during six hours (i.e, $\Delta t=1$ hour) and with a charging requirement of 9 $\mathrm{kWh}$ and maximum charging power of $3 \mathrm{~kW}$.

Table 1: Set of charging solutions of an EV offering upward reserve power in a six-hour availability period with a charging requirement of $9 \mathrm{kWh}$.

Solution (a), with constraint (7), is unfeasible because the charging requirement is already satisfied after interval $\mathrm{H} 3$, and the aggregator makes an upward reserve offer in intervals $\mathrm{H} 5$ and $\mathrm{H} 6$ where it is not able to supply if requested by the TSO.

Solution (b) is feasible. For instance, in interval $\mathrm{H} 3$ the EV offers $3 \mathrm{~kW}$ of upward reserve, and it consumes additional $3 \mathrm{~kW}$ in the remaining time intervals ( $\mathrm{H} 4$ in this case).

It is important to stress that constraint (7) offers robust solutions since the available reserve power in the current interval is not affected even if the upward reserve is dispatched in lower quantities during previous intervals. For instance, if the reserve in interval H1 of solution (b) is not fully dispatched, there would be a surplus of consumed electrical energy compared to what was planned, but the aggregator can consume less in interval H2 (if necessary) to compensate this surplus at a cost of an energy imbalance penalty.

The reserve band is divided into upward and downward directions with the following equality:

$$
P_{t, j}^{u p}=\mu \cdot P_{t, j}^{\text {down }}, \forall j \in\left\{1, \cdots, M_{t}\right\}, \forall t \in H,
$$

In the Iberian market, the reserve band is divided into $2 / 3$ for upward and $1 / 3$ for downward, so the value of $\mu$ is 2 . In markets without a rule for splitting the reserve band, the value of $\mu$ can be defined by considering the reserve prices, or the reserve reliability (e.g., estimate a $\mu$ from historical data that leads to the minimum reserve shortage), or a trade-off between both criteria.

The optimization problem of Equations (1)-(8) is an LP problem that can be solved using any commercial or non-commercial LP solvers.

After, solving the LP problem, a post-processing phase is applied to the downward reserve band. In order to create sufficient flexibility for supplying upward reserve, the purchased energy is higher than the charging requirement [see equation (2)]. Thus, a post-processing phase is necessary to eliminate downward reserve bids from the time intervals where the total purchased energy is above the charging requirement. This is performed with the values of $E_{t, j}$ calculated by solving the LP problem and with the following equation: 


$$
P_{t, j}^{\text {down }}=\left\{\begin{array}{c}
\min \left(\hat{R}_{j}-\sum_{k=t_{\text {tintal }}}^{t}\left(E_{k, j}\right), P_{t, j}^{d o w n}\right), \text { if } P_{t, j}^{d o w n} \cdot \Delta t+\sum_{k=t_{\text {tintial }}}^{t}\left(E_{k, j}\right) \leq \hat{R}_{j} \\
0, \text { if } P_{t, j}^{\text {down }} \cdot \Delta t+\sum_{k=t_{\text {initial }}}^{t}\left(E_{k, j}\right)>\hat{R}_{j}
\end{array}\right.
$$

where $t_{\text {initial }}$ is the first time interval of the availability period, i.e. $t_{\text {initial }} \in \hat{H}_{j}^{\text {plug }}$.

After adjusting the downward reserve band, the upward reserve band is also adjusted with equality (8).

Equation (9) increases the robustness of the downward reserve bid since, even in cases where the upward reserve from the previous intervals is not dispatched, the aggregator is able to supply the downward power in the subsequent intervals regardless of the dispatched upward reserve.

Table 2 presents a potential solution for energy and reserve bids of one EV with charging requirement of $9 \mathrm{kWh}$. In this example, the downward reserve power bid in interval $\mathrm{H} 5$ is removed in the postprocessing phase, since the sum of $E_{k}$ between intervals $\mathrm{H} 1$ and $\mathrm{H} 4$ is already equal to the charging requirement. Therefore, there is a risk that the EV may not be able to make available a downward reserve power of $1 \mathrm{~kW}$ in interval $\mathrm{H} 5$. For instance, if in interval $\mathrm{H} 2$ only $0.5 \mathrm{kWh}$ is dispatched as upward reserve and in interval $\mathrm{H} 3$ only $0.2 \mathrm{kWh}$, the total electrical energy after interval $\mathrm{H} 4$ would be $8.3 \mathrm{kWh}$, and, since it can only charge additional $0.7 \mathrm{kWh}$, the $\mathrm{EV}$ is unable to guarantee a downward reserve power of $1 \mathrm{~kW}$ in interval $\mathrm{H} 5$. In this case, the aggregator only offers downward reserve during the first four intervals.

Table 2: Example of a charging solution of an EV offering upward and downward reserve power in a six-hour availability period with a charging requirement of $9 \mathrm{kWh}$.

\section{Operational Management Algorithm}

The previous section described the day-ahead optimization model for deriving the energy and secondary reserve bids. During the operating day, the aggregator coordinates the EV charging to comply with the AGC signal and deliver secondary reserve with acceptable reliability. This section describes an operational management algorithm to meet this goal. This algorithm is divided into two phases: first, the redefinition of the EV fleet operating point and the calculation of the available reserve power (section 4.1), and then, the coordination of the EV charging to comply with the AGC requests (section 4.2).

\subsection{Redefinition of the Operating Point and Calculation of the Available Reserve Power}

The aggregator, 15 minutes before the beginning of time interval $t_{0}$ (e.g., necessary time to activate tertiary or balancing reserve if necessary), using the information from the plugged-in EV (i.e., communicated target SOC and expected departure hour), calculates the available reserve power in both 
directions for that interval and communicates this information to the SO. These values are updated during the operation hour since the available reserve power can be reduced if the reserve is dispatched in one direction during a long period.

The first step for computing the available reserve consists in determining the operating point $P_{t 0}^{\prime}$ of the EV fleet. The operating point is a constant charging level that the aggregator can sustain during the complete interval $t_{0}$ by coordinating the EV fleet charging and from which the upward and downward reserves are supplied.

Without the presence of uncertainty, the operating point would be equal to the accepted energy bid. However, because of forecast errors, the operating point will deviate from the energy bid, which creates energy imbalances and decreases the availability of secondary reserve. Therefore, the aggregator should define an operating point during the operational phase that guarantees the contracted reserve at a cost of increasing the energy imbalances. The following paragraphs describe a procedure that re-calculates the operating point (using the energy bid as reference), in order to maximize the availability of secondary reserve.

First, the aggregator, before the beginning of time interval $t_{0}$, and using the information of all pluggedin EV, computes two variables: $\bar{P}_{t_{0}}^{\text {min }}$, minimum, constant and feasible charging power of the EV fleet in time interval $t_{0} ; \bar{P}_{t_{0}}^{\max }$, maximum, constant and feasible charging power of the EV fleet in time interval $t_{0}$. The value of $\bar{P}_{t_{0}}^{\text {min }}$ is computed by solving an LP optimization problem with the following objective function:

$$
\min \left\lfloor\varphi\left(\sum_{j=1}^{M_{t_{0}}}\left(E_{t_{0}, j}^{*}\right)-0\right)+\sum_{k=t_{0}+1}^{T}\left(\varphi\left(E_{k}-\sum_{j=1}^{M_{k}}\left(E_{k, j}^{*}\right)\right)\right)\right\rfloor
$$

where $E_{k, j}{ }^{*}$ is the decision variable and corresponds to the actual energy consumed by the $\mathrm{j}^{\text {th }} \mathrm{EV}, t_{0}$ is the first time interval of the optimization period, $E_{k}$ is the result (or accepted bid) from the day-ahead optimization model, $\varphi$ is a piecewise loss function and $T$ is the time interval of the last plugged-in EV to depart. The loss function $\varphi$ is a convex function with the following form:

$$
\varphi(u)=\left\{\begin{array}{c}
u \cdot \hat{\pi}_{k}^{+}, u \geq 0 \\
-u \cdot \hat{\pi}_{k}^{-}, u<0
\end{array}\right.
$$

where $\pi_{k}^{+}$and $\pi_{k}^{-}$are the forecasted penalization prices for positive and negative deviations respectively. These two prices are forecasted with the Holt-Winters model for irregular time series [23]. This convex function can be converted into a linear function by using its epigraph form [24]. 
The constraints of the optimization problem are:

- the total energy consumed during the availability period must be equal to the charging requirement:

$$
\sum_{k \in H_{j}^{\text {plug }}}\left(E_{k, j}^{*}\right)=R_{t_{0}, j}, \forall j \in\left\{1, \cdots, M_{t}\right\}, \forall k \in H_{j}^{\text {plug }}
$$

where $R_{t 0, j}$ is the residual charging requirement (calculated from the communicated target SOC) at the beginning of time interval $t_{0}, H_{j}^{\text {plug }}$ is the availability period of the $j^{\text {th }} \mathrm{EV}$ (calculated from the communicated expected departure hour).

- the consumed energy in each time interval must be below or equal to the maximum available power for charging:

$$
E_{k, j}^{*} / \Delta t \leq P_{j}^{\max }, \forall j \in\left\{1, \cdots, M_{t}\right\}, \forall k \in H_{j}^{\text {plug }}
$$

This optimization problem consists in charging the EV fleet as close as possible to zero in time interval $\mathrm{t}_{0}$, and the value of $\bar{P}_{t_{0}}^{\min }$ is given by $\sum_{j=1}^{M_{t_{0}}}\left(\frac{E_{t_{0}, j}^{*}}{\Delta t}\right)$.

The value of $\bar{P}_{t_{0}}^{\max }$ is calculated with:

$$
\overline{\mathrm{P}}_{\mathrm{t}_{0}}^{\max }=\sum_{j=1}^{M_{t_{0}}}\left(\min \left(\frac{R_{t_{0}, j}}{\Delta t}, P_{j}^{\max }\right)\right)
$$

which means that it is equal to the maximum charging power constrained by the residual charging requirement. For instance, an EV with charging requirement equal to $1 \mathrm{kWh}$ in a half-hour period and with a maximum charging power of $3 \mathrm{~kW}$ can only charge at constant $2 \mathrm{~kW}\left(=\overline{\mathrm{P}}_{\mathrm{t}_{0}}^{\max }\right)$ during that interval.

These two variables, together with the accepted energy bid $\left(E_{t 0}\right)$, are used to define the operating point. Figure 4 depicts three situations that may occur in terms of energy bid value and the variables required to calculate the EV fleet operating point.

\section{Figure 4: Variables required to redefine the operating point of the EV fleet.}

In situation (a), the accepted energy bid is within the minimum and maximum consumption power limits. In order to guarantee full availability of the reserve power, the operating point should be within two limits: upper power limit that guarantees full availability of downward reserve power in time interval $t_{0}\left(\bar{P}_{t_{0}}^{\text {upper }}=\bar{P}_{t_{0}}^{\text {max }}-P_{t_{0}}^{\text {down }}\right)$, and lower power limit that guarantees full availability of upward reserve 
power $\left(\bar{P}_{t_{0}}^{\text {lower }}=\bar{P}_{t_{0}}^{\min }+P_{t_{0}}^{u p}\right)$. Depending on the accepted energy bid value, the following can occur:

- if $\bar{P}_{t_{0}}^{\text {upper }} \geq \bar{P}_{t_{0}}^{\text {lower }}$ (any operating point between $\bar{P}_{t_{0}}^{\text {lower }}$ and $\bar{P}_{t_{0}}^{\text {upper }}$ allows full availability of the reserve, thus it is selected the closest point to the energy bid)

- if $E_{t_{0}} / \Delta t \in\left[\bar{P}_{t_{0}}^{\text {lower }}, \bar{P}_{t_{0}}^{\text {upper }}\right] \Rightarrow P_{t_{0}}^{\prime}=E_{t_{0}} / \Delta t$

- if $\bar{P}_{t_{0}}^{\text {lower }}>E_{t_{0}} / \Delta t \Rightarrow P_{t_{0}}^{\prime}=\bar{P}_{t_{0}}^{\text {lower }}$ (the operating point is made equal to $\bar{P}_{t_{0}}^{\text {lower }}$ since it is the closest point to the energy bid)

- if $\bar{P}_{t_{0}}^{\text {upper }}<E_{t_{0}} / \Delta t \Rightarrow P_{t_{0}}^{\prime}=\bar{P}_{t_{0}}^{\text {upper }}$ (the operating point is made equal to $\bar{P}_{t_{0}}^{\text {upper }}$ since it is the closest point to the energy bid)

- if $\bar{P}_{t_{0}}^{\text {upper }}<\bar{P}_{t_{0}}^{\text {lower }}, P_{t_{0}}^{\prime}=E_{t_{0}} / \Delta t$ (any change in the operating point value would increase the reserve availability in one direction, at the cost of the other direction; the choice is to maintain the operating point equal to the energy bid value)

In situation (b), the accepted energy bid is below the minimum consumption power level. The operating point is defined as follows:

- if $\bar{P}_{t_{0}}^{\text {upper }} \geq \bar{P}_{t_{0}}^{\text {lower }} \Rightarrow P_{t_{0}}^{\prime}=\bar{P}_{t_{0}}^{\text {lower }}$ (the operating point is made equal to $\bar{P}_{t_{0}}^{\text {lower }}$ since it is the closest point to the energy bid);

- if $\bar{P}_{t_{0}}^{\text {upper }}<\bar{P}_{t_{0}}^{\text {lower }}, P_{t_{0}}^{\prime}=\min \left(\bar{P}_{t_{0}}^{\text {upper }}, \bar{P}_{t_{0}}^{\text {min }}\right)$ (it is not possible to offer the full contracted reserve in both directions; if $\bar{P}_{t_{0}}^{\text {upper }}$ is greater than $\bar{P}_{t_{0}}^{\text {min }}$, it is not possible to offer upward reserve power and the operating point is made equal to $\bar{P}_{t_{0}}^{\text {min }}$; if it is lower, the operating point is made equal to $\bar{P}_{t_{0}}^{\text {upper }}$ and it is possible to offer upward reserve between this point and $\bar{P}_{t_{0}}^{\text {min }}$ ).

In situation (c), the accepted energy bid is greater than the maximum consumption power level. The operating point is defined as follows:

- if $\bar{P}_{t_{0}}^{\text {upper }} \geq \bar{P}_{t_{0}}^{\text {lower }} \Rightarrow P_{t_{0}}^{\prime}=\bar{P}_{t_{0}}^{\text {upper }}$ (the operating point is made equal to $\bar{P}_{t_{0}}^{\text {upper }}$ since it is the closest point to the energy bid);

- if $\bar{P}_{t_{0}}^{\text {upper }}<\bar{P}_{t_{0}}^{\text {lower }}, P_{t_{0}}^{\prime}=\min \left(\bar{P}_{t_{0}}^{\text {lower }}, \bar{P}_{t_{0}}^{\text {max }}\right)$ (it is not possible to offer the full contracted reserve in both directions; if $\bar{P}_{t_{0}}^{\text {lower }}$ is greater than $\bar{P}_{t_{0}}^{\max }$, it is not possible to offer downward reserve power and the operating point is made equal to $\bar{P}_{t_{0}}^{\max }$; if it is lower, the operating point is made 
equal to $\bar{P}_{t_{0}}^{\text {lower }}$ and it is possible to offer downward reserve between this point and $\bar{P}_{t_{0}}^{\max }$ ).

480 The goal of this approach was to change the operating point in order to comply with the contracted reserve power, while at the same time, it tries to avoid a significant increase of energy imbalances. This change in the operating point creates an energy imbalance which the TSO solves by calling balancing or tertiary reserve, and the aggregator pays a financial penalty for this energy imbalance. available upward reserve power $\left(P_{t_{0}}^{u p}\right)$ is given by:

$$
P_{t_{0}}^{u p}=\min \left(P_{t_{0}}^{\prime}, P_{t_{0}}^{u p}\right)
$$

This equation means that the aggregator can only decrease a charging rate that is attainable. For instance, if the upward reserve bid is $5 \mathrm{MW}$ and the operating point is only $3 \mathrm{MW}$, then the available reserve capacity should be $3 \mathrm{MW}$.

The available downward reserve power $\left(P_{t_{0}}^{\text {down }}\right.$ ) is given by:

$$
P_{t_{0}}^{\text {down }}=\min \left(P_{t_{0}}^{\text {down }}, \sum_{j \in K}\left(P_{j}^{\max }\right)-P_{t_{0}}^{\prime}\right)
$$

where $K$ is the set of plugged-in EV in $t_{0}$ with $R_{t 0}>0$ (i.e., the charging requirement is not fully

The available reserve power is the minimum between accepted bid and the difference between the maximum instantaneous charging power of the EV fleet and the operating point.

It may happen that both $P_{t_{0}}^{\prime u p}$ and $P_{t_{0}}^{\prime d o w n}$ become depleted after some time $(<\Delta t)$, and in this case, the secondary reserve is replaced by the tertiary reserve. Note that this does not jeopardize the power system security since the SO, in order to replace this depleted reserve (i.e., free up additional secondary reserve), calls tertiary reserve, which is translated into an increasing use of tertiary reserve.

The stochastic nature of the EV behavior contributes to this reserve depletion, but it should be underlined that EV supply reserve within the battery energy constraints and the driver's preferences. For instance, in upward reserve provision, since the main priority is to satisfy the charging requirement of the EV drivers, it may not be possible to reduce the charging rate for a long period of time. For the downward reserve, reserve depletion happens when the batteries of some EV become full during $\Delta t$. The same problem is valid for storage devices and other controllable loads. 
The aggregator, in the beginning of time interval $t_{0}$, dispatches the $\mathrm{EV}$ for consuming the operating point $\left(P_{t 0}^{\prime}\right)$ in $t_{0}$ and minimizing the deviations to the accepted energy bids (in $\left.k>t_{0}\right)$. This is accomplished with the following objective function as follows:

$$
\min \left[\varphi\left(P_{t_{0}}^{\prime}-\sum_{j=1}^{M_{t_{0}}}\left(\frac{E_{t_{0}, j}^{*}}{\Delta t}\right)\right)+\sum_{k=t_{0}+1}^{T}\left(\varphi\left(E_{k}-\sum_{j=1}^{M_{k}}\left(E_{k, j}^{*}\right)\right)\right)\right]
$$

The two constraints (11) and (12) are also considered.

When upward reserve is needed, the AGC sends a signal to the aggregator, and the aggregator dispatches the EV to supply the requested reserve using the following objective function:

$$
\min \left[\varphi\left(\left(P_{t_{0}}^{\prime}-P_{t_{0}}^{\prime \text { up }}\right)-\sum_{j=1}^{M_{t_{0}}}\left(\frac{E_{t_{0}, j}^{*}}{\Delta t^{\prime}}\right)\right)+\sum_{k=t_{0}+1}^{T}\left(\varphi\left(E_{k}-\sum_{j=1}^{M_{k}}\left(E_{k, j}^{*}\right)\right)\right)\right]
$$

where $\Delta t^{\prime}(\leq \Delta t)$ is the length of the period where the secondary reserve was activated (i.e., equivalent number of minutes).

When the AGC sends a signal requesting downward reserve, the following objective function is used:

$$
\min \left[\varphi\left(\left(P_{t_{0}}^{\prime}+P_{t_{0}}^{\prime d o w n}\right)-\sum_{j=1}^{M_{t_{0}}}\left(\frac{E_{t_{0}, j}^{*}}{\Delta t^{\prime}}\right)\right)+\sum_{k=t_{0}+1}^{T}\left(\varphi\left(E_{k}-\sum_{j=1}^{M_{k}}\left(E_{k, j}^{*}\right)\right)\right)\right]
$$

The constraints (11) and (12) are also considered for these two objective functions.

This optimization problem can be solved in real-time, using any commercial or non-commercial LP solver, with an average execution time below one second (Intel Core i5 CPU M450 @ $2.40 \mathrm{GHz}$ processor and $4 \mathrm{~GB}$ of RAM and for $1500 \mathrm{EV}$ ).

As mentioned before, in some cases after supplying reserve during some time, the optimization problem may become unfeasible because of constraint (11), and the reserve is considered to be depleted. In this case, the aggregator communicates the new available reserve power to the SO, which can mobilize tertiary reserve to free up additional secondary reserve or dispatch additional reserve power from other resources. The aggregator incurs in a financial penalization for not being able to supply the required reserve (topic discussed in section 5).

The operational management algorithm is sequential and can be summarized as follows:

1. new information is available from the recently plugged-in EV (i.e., that connected for charging between $t_{0^{-}} 1$ and $t_{0}$ ) and is included in equation (11) of the optimization model; 
2. using this information, the aggregator computes the operating point, available upward and downward reserve power: $\mathrm{P}_{\mathrm{t} 0}^{\prime}, P_{t_{0}}^{\prime \text { up }}$ and $P_{t_{0}}^{\prime \text { down }}$ [equation (11)]. This information is communicated to the SO;

3. during time interval $t_{0}$ :

○ the AGC sends signals requesting upward or downward reserve. The aggregator solves the optimization problem from (18)-(19) and sends set points to the EV fleet. The prices $\pi_{\mathrm{t} 0}{ }^{+}$and $\pi_{\mathrm{t} 0}{ }^{-}$are made equal to a large number (e.g., $10^{3}$ );

- the aggregator updates the residual charging requirement of each EV based on the operating point plus dispatched reserve $\left(R_{t o+1, j}=R_{t 0, j} E_{t 0, j}{ }^{*}\right)$. Moreover, it updates and communicates the new values of available reserve to the SO;

4. this process is repeated for the next time interval $t_{0}+l$ with the recently arrived EV.

\section{Market Settlement}

After the operating day, there is a settlement phase where the penalty costs related to deviation from the purchased energy and reserve shortage are added to the cost from purchasing energy and to the income from having available reserve capacity. Some electricity markets already have penalties for reserve shortage [25][26], and four different penalization schemes are discussed in [13].

In this paper, two alternative penalization schemes for reserve shortage are considered: (1) the aggregator is penalized when it is unable to supply the full reserve capacity during the complete interval $\Delta t$ (based on the scheme adopted in Portugal [25]); (2) the aggregator is only penalized when it fails to respond with sufficient reserve capacity to a signal from the AGC (based on a scheme proposed in [13]).

For settlement scheme (1), in each direction of reserve, the aggregator is penalized by the difference between the accepted reserve bid and the reserve power that can be sustained during the complete interval $t$. The downward reserve power that can be sustained during interval $t_{0}\left(\bar{P}_{t_{0}}^{\text {down }}\right)$ is given by:

$$
\bar{P}_{t}^{\text {down }}=\min \left(P_{t}^{\text {down }}, \bar{P}_{t}^{\max }-P_{t}^{\prime}\right)
$$

For the upward reserve, the $\bar{P}_{t_{0}}^{u p}$ is given by:

$$
\bar{P}_{t}^{u p}=\min \left(P_{t}^{u p}, P_{t}^{\prime}-\bar{P}_{t}^{\min }\right)
$$

The system operator and regulator may audit these values to avoid fraud.

The total cost has the following terms: 


$$
\text { Total Cost }=\sum_{t}\left(\begin{array}{c}
P_{t}^{\prime} \cdot \Delta t \cdot p_{t}+P_{t}^{\prime d o w n} \cdot \lambda_{t}^{\text {down }} \cdot p_{t}^{\text {down }}- \\
P_{t}^{\text {upp }} \cdot \lambda_{t}^{u p} \cdot p_{t}^{u p}-p_{t}^{\text {cap }} \cdot\left(\bar{P}_{t}^{\text {down }}+\bar{P}_{t}^{u p}\right) \\
\Psi\left(P_{t}^{\prime} \cdot \Delta t, E_{t}\right)+\Phi\left(P_{t}^{\text {down }}, P_{t}^{\text {up }}, \bar{P}_{t}^{\text {down }}, \bar{P}_{t}^{u p}\right)
\end{array}\right)
$$

where $\lambda_{t}^{\text {down }}$ and $\lambda_{t}^{u p}$ are the number of equivalent minutes of dispatched downward and upward secondary reserve, $P_{t}{ }_{t} . \Delta t . p_{t}$ is the cost of energy consumed by the EV (i.e., operating point paid at the energy price), $P_{t}^{\text {down }} . \lambda_{t}^{\text {down }} \cdot p_{t}^{\text {down }}$ is the consumption corresponding to the dispatched downward secondary reserve, $P_{t}^{\prime u p} \cdot \lambda_{t}^{u p} \cdot p_{t}^{u p}$ is the income from dispatched upward reserve, $p_{t}^{\text {cap }} \cdot\left(\bar{P}_{t}^{\text {down }}+\bar{P}_{t}^{u p}\right)$ is the income from having available reserve capacity, $\Psi$ are the costs associated to deviations from the purchased energy (i.e. deviation between $E_{t}^{\text {cons }}$ and $E_{t}$ ), $\Phi$ are the costs associated to reserve shortage.

The cost term $\Psi$ works as follows: when the aggregator has surplus of energy it has to sell this extra at a regulation price $\left(p_{t}^{\text {surplus }}\right)$, in general below the energy price; if the situation is shortage of energy, it has to pay a regulation price $\left(p_{t}^{\text {shortage }}\right)$, in general above the energy price. This is translated into the following:

$$
\Psi=\left\{\begin{array}{l}
\left(E_{t}-P_{t}^{\prime} \cdot \Delta t\right) \cdot\left(p_{t}-p_{t}^{\text {surplus }}\right), E_{t}>P_{t}^{\prime} \cdot \Delta t \\
\left(P_{t}^{\prime} \cdot \Delta t-E_{t}\right) \cdot\left(p_{t}^{\text {shortage }}-p_{t}\right), E_{t} \leq P_{t}^{\prime} \cdot \Delta t
\end{array}\right.
$$

where the price difference $p_{t}-p_{t}^{\text {surplus }}$ is the positive deviations price $\left(\pi_{t}^{+}\right)$, and the difference $p_{t}^{\text {shortage }}-p_{t}$ is the negative deviations price $\left(\pi_{t}{ }^{-}\right)$.

In terms of reserve income, the aggregator is paid for the available reserve capacity and a penalty term proportional to the deviation between $P_{t}^{u p}$ and $\bar{P}_{t}^{u p}$ (and between $P_{t}^{\text {down }}$ and $\bar{P}_{t}^{\text {down }}$ ) is imposed. The penalty term $\Phi$ is as follows:

$$
\Phi=\left\{\begin{array}{c}
\alpha \cdot p_{t}^{\text {cap }} \cdot\left(P_{t}^{\text {down }}-\bar{P}_{t}^{\text {down }}\right), P_{t}^{\text {down }}>\bar{P}_{t}^{\text {down }} \\
\alpha \cdot p_{t}^{\text {cap }} \cdot\left(P_{t}^{\text {up }}-\bar{P}_{t}^{u p}\right), P_{t}^{\text {up }}>\bar{P}_{t}^{u p}
\end{array}\right.
$$

where $\alpha$ is a penalization coefficient that takes value 1.5 in this paper (i.e., value used in Portugal and Spain).

In settlement scheme (2), the total cost is given by:

$$
\text { Total Cost }=\sum_{t}\left(\begin{array}{c}
P_{t}^{\prime} \cdot \Delta t \cdot p_{t}+P_{t}^{\prime \text { down }} \cdot \lambda_{t}^{\text {down }} \cdot p_{t}^{\text {down }}- \\
P_{t}^{\prime \text { up }} \cdot \lambda_{t}^{\text {up }} \cdot p_{t}^{\text {up }}-p_{t}^{\text {cap }} \cdot\left(P_{t}^{\text {down }}+P_{t}^{\prime \text { up }}\right) \\
\Psi\left(P_{t}^{\prime} \cdot \Delta t, E_{t}\right)+\Phi\left(P_{t}^{\text {down }}, P_{t}^{\text {up }}, P_{t}^{\prime \text { down }}, P_{t}^{\prime \text { up }}, R N S_{t}^{\text {up }}, R N S_{t}^{\text {down }}\right)
\end{array}\right)
$$

$$
\text { Note that in this case the reserve capacity payment is a function of } P_{t}^{\prime d o w n} \text { and } P_{t}^{\prime \text { up }} \text {. Furthermore, the }
$$
reserve shortage penalty $\Phi$ has two components: one that penalizes the unavailable reserve capacity using 
equation (24), but for the deviation between $P_{t}^{u p}$ and $P_{t}^{\prime \text { up }}$ (and between $P_{t}^{\text {down }}$ and $P_{t}^{\prime d o w n}$ ); and another that penalizes the reserve not supplied (i.e., depleted reserve) $-R N S_{t}^{u p}$ and $R N S_{t}^{\text {down }}$. This gives the following:

$$
\begin{aligned}
& \Phi=\left\{\begin{array}{c}
\alpha \cdot p_{t}^{\text {cap }} \cdot\left(P_{t}^{\text {down }}-P_{t}^{\text {down }}\right), P_{t}^{\text {down }}>P_{t}^{\text {down }} \\
\alpha \cdot p_{t}^{\text {cap }} \cdot\left(P_{t}^{\text {up }}-P_{t}^{\text {up }}\right), P_{t}^{\text {down }}>P_{t}^{\text {down }}
\end{array}\right. \\
& +\gamma \cdot p_{t}^{\text {up }} \cdot\left(R N S_{t}^{\text {up }}\right)+\left(p_{t}-p_{t}^{\text {down }}\right) \cdot\left(R N S_{t}^{\text {down }}\right)
\end{aligned}
$$

where $\gamma$ is a penalization coefficient similar to $\alpha$. Inspired by the Demand Response Reserves Pilot Program at ISO New England [26], in this paper the value of $\gamma$ is made equal to one. For upward reserve, this means that the aggregator must supply more than $50 \%$ of the contracted reserve. Otherwise, the penalty term is greater than the payment for partially supplying the reserve. For the downward reserve, the penalization term is different. It is equal to the difference between $p_{t}^{\text {down }}$ and $p_{t}$, otherwise hours with $p_{t}^{\text {down }}$ equal to zero (i.e., expensive reserve hours) would not be penalized.

Finally, note that the prices $p_{t}^{u p}$ and $p_{t}^{\text {down }}$ are the prices of tertiary reserve in Portugal that is used to replace the depleted secondary reserve.

\section{Test Case Results}

\subsection{Description}

The test case uses electricity market data from two years (2010 and 2011) of the Iberian electricity market [27]. This market data consists of the following variables: market prices for energy; prices for available and dispatched secondary reserve; two binary variables indicating the direction of the dispatched secondary reserve; number of equivalent minutes of dispatched reserve of the thermal power plant of Lares (see Figure 1). The time interval length is half-hour $(\Delta t=30 \mathrm{~min})$.

Synthetic time series for the availability and consumption of 3000 battery EV along one year was simulated using a discrete-time-space Markov chain, in accordance with the traffic patterns in Portugal. The simulation time step is half-hour. Details about the simulation method can be found in [28].

\subsection{Sampling Process for Evaluation}

A sampling process based on [29] is used to generate 30 random repetitions of an evaluation experiment. The objective is to test the optimization models for different market data randomly sampled (but maintaining the temporal sequence) from the two-year period.

Since the forecasting algorithms require training and testing datasets, a fixed length for these two sets was defined: 9 months for training and 3 months for evaluation. Then, a sampling process without 
replacement is used to draw the first hour of the day, $x$, from the candidate set. This sample is used to split the three years of data in training (between $x$ and $x-9$ months) and evaluation (between $x$ and $x+3$ months) datasets. The process is repeated 30 times, and for each sample, the optimization models are tested in the evaluation dataset.

In order to test the optimization methodologies in different EV data, the synthetic time series for 3000 $\mathrm{EV}$ are divided into two groups with $1500 \mathrm{EV}$ : fleets A and B. The main difference between both fleets is that the drivers of fleet B drive more $\mathrm{km}$ on average. The battery size and consumption per km of both fleets are from the same database (i.e., have the same magnitude).

The following sampling process, based on the binary time series of the direction of dispatched secondary reserve in Portugal, is used to create different realizations of the number of equivalent minutes of dispatched secondary reserve:

- if upward secondary reserve is activated (i.e., the binary time series for upward reserve has value 1), a sample is taken from the distribution of the number of equivalent minutes from the histogram of Figure 1 (thermal power plant). This gives the value of $\Delta t^{\prime}$ in equation (19) and $\lambda_{t}^{u p}$ in (22);

- if downward secondary is activated, a sample is taken from the histogram for downward secondary reserve, and it gives the value of $\Delta t^{\prime}$ in equation (18) and $\lambda_{t}^{\text {down }}$ in (22);

- when the reserve is not dispatched in one direction, the values of $\Delta t^{\prime}$ and $\lambda$ are zero in that direction

\subsection{Illustrative Example}

Figure 5 depicts the output of the day-ahead optimization (section 3), the redefinition of the EV fleet operating point (section 4.1) and the output of the operational management algorithm (section 4.2) for one day (with hourly intervals) from the test case.

Figure 5: (a) Output of the day-ahead optimization (energy and secondary reserve power bids); (b) calculation of the redefined EV fleet operating point; (c) operating point, available upward and downward reserve power, and electrical energy consumed by the EV fleet during the operating interval.

In Figure 5a, the aggregator mostly presents reserve power bids in the period between hourly intervals 1 and 4 and intervals 20 and 24, while during the remaining intervals the offered reserve is rather low. Note that, in order to offer secondary reserve power, the aggregator must offer an energy bid that is the reference operating point from which supplies upward and downward reserve. For instance, in hour 2, the 
energy bid is $1.01 \mathrm{MW}$, from which a regulation power of $0.9 \mathrm{MW}$ is offered in upward direction (upward band is between 1.01 MW and $0.11 \mathrm{MW}$ ) and half of this value is offered in the downward direction (reserve band is between 1.01 MW and 1.46 MW).

This bids pattern is consistent with the drivers' behavior. The available power for secondary reserve is higher when the number of plugged-in EV is high and also when the charging requirements are not yet fully satisfied. For instance, in intervals 5 and 6 , the secondary reserve bid is zero, since either the charging requirement of the EV is almost satisfied or there is no flexibility to postpone charging, as the EV will depart in the next intervals. The aggregator offers upward power earlier (between intervals 19 and 24 and between 1 and 4 ) to consume after those intervals the necessary energy to meet the charging requirement.

The estimated total cost, calculated from the objective function (1), and assuming that the upward and downward reserve are $100 \%$ dispatched, is $182.7 €$. This cost is just an estimate and only after the operational phase is it possible to calculate the real wholesale total cost.

Figure 5b depicts the redefinition of the operating point, in which the accepted energy bid, the consumption limits, and the redefined operating point are depicted for each hour. The first grey area is the interval between $\bar{P}_{t_{0}}^{\text {min }}$ and $\bar{P}_{t_{0}}^{\text {max }}$, which defines the range of feasible values for the EV fleet charging power taking into account its constraints. The dark grey area is the interval between $\bar{P}_{t_{0}}^{\text {lower }}$ and $\bar{P}_{t_{0}}^{\text {upper }}$ which defines the range of charging power values that assure a compliance with the contracted secondary reserve levels.

All the operating points are within these two bands (which means no reserve power shortage), while the accepted bids in intervals 6,7 and 12 are above the limit $\bar{P}_{t_{0}}^{\max }$. Thus, in these three intervals the operating point cannot be equal to the accepted energy bid (i.e., it is lower). In interval 1, the accepted energy bid (that corresponds to $0.6 \mathrm{MW})$ is below the limit $\bar{P}_{t_{0}}^{\text {lower }}(0.655 \mathrm{MW})$, thus the operating point is made equal to the lower limit.

The operational algorithm concludes the management process of the EV fleet charging. The output (or result) of this last phase is the electrical energy consumed by the EV fleet in each hour, which is depicted in Figure 5c. The operating point and the available upward and downward reserve power are also depicted. For instance, in hour 1 , the number of equivalent minutes of dispatched reserve was 42.24 minutes for downward and 17.76 minutes for upward, and the electrical energy consumed by the EV fleet 
by the end of that interval was $0.68 \mathrm{MWh}$, which corresponded to increasing the charging level from 0.65 MW $\left(P_{t 0}^{\prime}\right)$ to $0.68 \mathrm{MW}$ by supplying more downward than upward reserve power. In hour 24 , the secondary reserve was also activated in both directions (46 minutes for upward and 8.5 minutes for downward reserve), and the consumed electrical energy was below the operating point.

The market settlement described in section 5 is applied a posteriori (i.e., after the operational management phase) and it gives the "true" cost of the aggregator. In this illustrative example, the total cost, after the operational management phase, was $204.9 €$ (in contrast to $182.7 €$ estimated the day before). This cost difference is explained by a dispatched reserve power below $100 \%$, price forecast errors, and imbalance costs.

\subsection{Results}

The participation of the two EV fleets in the energy and secondary reserve market was simulated for the test periods resulting from the sampling process. An evaluation of the forecast quality is presented in the appendix.

Figure 6a depicts the total cost reduction in fleets A and B [with settlement scheme (1)], using as reference the total cost from optimizing only the energy bid (i.e., no secondary reserve bids) with the divided approach described in [15]. The results for the 30 samples are presented with a boxplot.

Figure 6: (a) Total cost reduction in fleets $A$ and $B$ for settlement scheme (1), using the participation in the energy market as reference; (b) total cost reduction of scheme (2) compared to scheme (1).

The results for scheme (1) show that the participation in the secondary reserve market decreases the total cost in $31 \%$ for fleet $\mathrm{A}$ and $37.1 \%$ for fleet $\mathrm{B}$. These results show that the proposed optimization framework is able to provide a considerable cost reduction to the aggregator.

Figure 6b compares the total cost reduction of settlement scheme (2) compared to the total cost calculated with scheme (1). Scheme (2) penalizes less the situations with reserve shortage, since the aggregator only loses part of the income when it is not able to follow the AGC regulation signal. The cost reduction is higher in fleet $\mathrm{B}$, which, as it will be show in this section, is the case with the highest reserve shortage magnitude. Therefore, settlement scheme (2) is financially more attractive to the EV aggregator and creates more incentives for the EV participation since it takes into account the stochastic nature of the EV supplying secondary reserve. Scheme (1), from the TSO's viewpoint, is more attractive since it demands a higher compliance is terms of reserve provision (or penalizes more reserve shortage events). Nevertheless, since EV is a cheap and fast responding reserve resource, compared to the conventional 
ones, the TSO can adopt scheme (2) to better account for its specific characteristics.

In addition to evaluating the cost reduction, it is necessary to evaluate the magnitude of reserve shortage events that result from using the proposed optimization framework. This indicates, from the TSO's viewpoint, the degree of reliance on this reserve resource.

The percentage of reserve capacity shortage (pRCS) is computed as follows:

$$
p R C S=\frac{\sum_{t}\left(P_{t}^{u p}-P_{t}^{\prime u p}\right)}{\sum_{t}\left(P_{t}^{u p}\right)} \cdot 100 \%
$$

For the upward reserve, the pRCS on average is equal to $0.005 \%$ for fleet $\mathrm{A}$ and $0.0 \%$ for fleet $\mathrm{B}$. For the downward reserve, the pRCS on average is equal to $0.17 \%$ for fleet $\mathrm{A}$ and $2.42 \%$ for fleet $\mathrm{B}$.

For scheme (1), equation (27) can be used to compute the pRCS of the difference between $P_{t}^{\text {down }}-\bar{P}_{t}^{\text {down }}$

Figure 7 depicts the pRCS of upward reserve in fleets A and B for scheme (1) and for each SOC tolerance level. An additional option is tested when supplying upward reserve. In the contract between the driver and the aggregator, a degree of flexibility for the SOC is established. The aggregator only guarantees $95 \%$ or $90 \%$ of SOC (instead of $100 \%$ ) when there is a risk of upward reserve shortage. Thus, the pRCS results are presented for three possible SOC tolerance levels: 100\%, 95\% and 90\%.

With a $100 \%$ SOC, the average pRCS is $0.05 \%$ in fleet A and $1.19 \%$ in fleet B. In fleet B, when the SOC tolerance is $90 \%$, the pRCS presents a significant decrease, showing an average value of $0.01 \%$.

Figure 7: Percentage of reserve capacity shortage (pRCS) in fleets A and B.

To evaluate the upward reserve reliability under scheme (2), the percentage of upward reserve not supplied (pRNS) is depicted in Figure 8. In this case, and in contrast to scheme (1), the reserve shortage from not following an AGC signal is lower compared to Figure 7.

Figure 8: Percentage of upward reserve not supplied (pRNS) in fleets A and B.

It is important to underline that the different results obtained for each test sample are exclusively because of different realizations (or test samples) of the number of equivalent minutes of dispatched reserve, and because of the forecasted and realized market prices. These different realizations lead to distinct energy and secondary reserve bids, which ultimately lead to distinct results in terms of reserve shortage.

Figure 9 presents the pRCS and pRNS for the downward reserve. With exception of pRCS in fleet B, all cases show a low pRCS and pRNS meaning that the deviation between available and contracted 
reserve power is low. The higher values of pRCS in fleet B can be explained by the negative bias (overestimation) of the charging requirement forecast (see Table $\mathbf{3}$ in the appendix), which is translated into an overestimation of the actual charging values as shown in [31]. An overestimation of the charging requirement contributes to an overestimation of the downward reserve power and consequently to an increase of the reserve shortage due to forecast errors.

\section{Figure 9: pRCS and pRNS of downward reserve in fleets $A$ and $B$.}

Finally, Figure 10 depicts the cost reduction for two cases: perfect forecast for the EV variables used in the day-ahead optimization; perfect forecast for all variables. The reference for computing the cost reduction is the energy and secondary reserve bids with forecasts for all the variables [i.e., the result from Figure 6b using scheme (2)].

The use of perfect forecasts for the EV variables only accomplishes a cost reduction of $4.1 \%$ in fleet A and $6.7 \%$ in fleet B on average. This suggests that the uncertainty of the EV variables has a small impact in the total cost. The impact on cost reduction is substantial when perfect forecasts are used for all the variables (e.g., market prices, dispatched reserve): $71.5 \%$ in fleet A and $67.1 \%$ in fleet B. Nevertheless, this "perfect forecast" assumption is only theoretical since variables, such as the reserve direction, cannot be forecasted with acceptable accuracy (as discussed in section 2.2), which shows that neglecting this uncertainty may lead to very optimistic results.

Figure 10: Reduction in the total cost for both fleets with two different sets of available information: perfect forecast for the EV variables; perfect forecast for all the variables. The reference is the result obtained with forecasts for all the variables.

\section{Conclusions}

This paper presents a new optimization model for energy and secondary reserve bids in the day-ahead market. Moreover, following the day-ahead bidding, a new operational management algorithm that coordinates the EV charging for minimizing the difference between contracted and realized values is also described. Using the day-ahead and operational algorithm, the total wholesale cost of the EV aggregator decreased on average between $30 \%$ and $35 \%$, compared to a strategy that only optimizes the energy bids. The algorithms are also capable of assuring the contracted reserve with acceptable reliability (e.g., the percentage of reserve capacity shortage ranges between $0 \%$ and $1.8 \%$ on average). This high reliability is important from the TSO's viewpoint.

The results show that the role of the forecast errors in the market and EV variables cannot be 
neglected. These forecast errors create deviations between purchased and consumed energy and impact the reliability of the reserve provision. The assumption of perfect forecast, or the incorrect evaluation of the algorithm performance (e.g., neglecting the need to calculate the true available reserve power), might lead to excessively optimistic results.

The presence of reserve shortage events emphasizes the importance of defining suitable market rules and protocols for creating financial incentives to avoid these situations. Nevertheless, at the same time, these rules should take into account the stochastic nature of the EV behavior. To overcome this problem, several SO in the USA are adopting two solutions, which are briefly described here.

The first solution consists in designing a new AGC control signal (in addition to the traditional signal) to improve the participation of fast responding resources (e.g., flywheels, batteries) [31]. The direction of this new signal changes rapidly in order to ensure a short-term net energy around zero after a short period (e.g., 5 minutes). This means that EV can supply upward and downward reserve power during each operating period without any energy constraints related to the depth of discharge or maximum storage capacity.

The second solution follows FERC (Federal Energy Regulatory Commission) Order 755 and consists in creating a performance score that rewards resources that provide reserve more quickly. For instance, the score created by PJM includes a component that measures the difference between the energy the SO requests and how much the resource provides (i.e., penalizes reserve shortage), but also a component measuring the delay (i.e., ramp capability) in the reserve response [31]. In this case, the EV aggregator can present a lower performance in the precision component compared to conventional resources, but on the other hand, presents a higher performance in the delay component.

It is important to underline that even with the risk of reserve shortage, the EV aggregator is an important asset to the SO since it provides fast-responding reserve, it is a resource that already exists in the system (i.e., it does not require incentives for investment), and it is greenhouse gas emissions free compared to conventional power plants.

Finally, the optimization framework proposed in this paper can be adapted to other type of flexible loads (e.g., electric boilers). Topics for future research are the participation in intraday markets and the inclusion of probabilistic information of prices, and EV variables in the day-ahead optimization model.

\section{Appendix - Forecast Quality}

Table 3 presents the mean absolute percentage error (MAPE) and percentage bias (PBIAS) of the 
availability and charging requirement forecasts for the whole EV fleet (i.e., sum of the individual forecasts for each EV). A detailed evaluation of the forecasts for the EV availability and charging requirement can be found in [30] for these two fleets.

Table 3: MAPE and PBIAS of the aggregated availability and charging requirement forecast of fleets A and B.

A detailed evaluation of the forecasts for the EV availability and charging requirement can be found in [30] for these two fleets.

Table 4 presents the mean absolute error (MAE) and root mean square error (RMSE) of the forecasted energy and reserve prices.

Table 4: MAE and RMSE of the forecasted energy and reserve prices (average values of 30 samples).

\section{Acknowledgements}

The work of R. J. Bessa was supported by FCT - Fundação para a Ciência e a Tecnologia (Portuguese Foundation for Science and Technology) Ph.D. Scholarship SFRH/BD/33738/2009 and by National funds through the FCT within project «SMAGIS - PTDC/SEN-ENR/113094/2009».

The authors acknowledge Filipe J. Soares from INESC TEC to provide the electric vehicles synthetic time series data used in this paper.

[1] G. Heffner, C. Goldman, B. Kirby, M. Kintner-Meyer, Loads providing ancillary services: review of international experience, Tech. Report, Lawrence Berkeley National Laboratory (2007).

[2] D.S. Callaway, I.A. Hiskens, Achieving controllability of electric loads, Proc. of the IEEE 99 (2011) 184-199.

[3] R.J. Bessa, M.A. Matos, Economic and technical management of an aggregation agent for electric vehicles: a literature survey, Eur. Tran. on Elect. Power 22 (2012) 334-350.

[4] C. Guille, G. Gross, A conceptual framework for the vehicle-to-grid (V2G) implementation, Energy Policy 37 (2009) 4379-4390.

[5] J.A. Peças Lopes, F.J. Soares, P.R.R. Almeida, Integration of electric vehicles in the electric power system, Proc. of the IEEE 99 (2011) 168-183.

[6] P.M. Almeida, Impact of vehicle to grid in the power system dynamic behavior, PhD Thesis, Faculty of Engineering from the University of Porto (2011).

[7] A. Brooks, S.H. Thesen, PG\&E and Tesla Motors: vehicle to grid demonstration and evaluation program, Proc. of the 23rd Inter. Bat., Hyb. and Fuel Cell Elect. Veh. Symp. \& Exh. (2007).

[8] E. Sortomme, M.A. El-Sharkawi, Optimal charging strategies for unidirectional vehicle-to-grid, IEEE Trans. on Smart Grid 2 (2011) 119-126.

[9] Sekyung Han, S. Han, K. Sezaki, Development of an optimal vehicle-to-grid aggregator for frequency regulation, IEEE Trans. on Smart Grid 1(2010) 65-72. 

Trans. on Power Sys. 26 (2011) 1021-1029.

[11] C. Wu, H. Mohsenian-Rad, J. Huang, Vehicle-to-aggregator interaction game, IEEE Trans. on Smart Grid 3 (2012) 434442.

[12] M. Pantos, Exploitation of electric-drive vehicles in electricity markets, IEEE Trans. on Power Sys. 27 (2012) 682-694.

[13] Sekyung Han, S. Han, and K. Sezaki, Estimation of achievable power capacity from plug-in electric vehicles for V2G frequency regulation: case studies for market participation, IEEE Trans. on Smart Grid 2 (2011) 632-641.

[14] R.J. Bessa, M.A. Matos, F.J. Soares, J.A. Peças Lopes, Optimized bidding of a EV aggregation agent in the electricity market, IEEE Trans. on Smart Grid 3 (2012) 443-452.

[15] R.J. Bessa, M.A. Matos, Global against divided optimization for the participation of an EV aggregator in the day-ahead electricity market - part I: theory, Elect. Power Sys. Res. 95 (2013) 319-329.

[16] R.J. Bessa, M.A. Matos, Optimization models for EV aggregator participation in a manual reserve market, IEEE Trans. on Pow. Sys. (2013) in press.

[17] E.L. Miguélez, I.E. Cortés, L. Rouco, G. López, An overview of ancillary services in Spain, Elec. Power Sys. Res. 78 (2008) 515-523

[18] J. Rogers and K. Porter, Wind power and electricity markets, Technical Report, Utility Wind Integration Group (2011).

[19] Operational handbook. P1 - Policy 1: Load-Frequency Control and Performance, ENTSO-E (2009). [Online] http://www.entsoe.eu/resources/publications/system-operations/operation-handbook/ (accessed in February 2013)

[20] E. Hirst, Integrating wind output with bulk power operations and wholesale electricity markets, Wind Energy 5 (2002) 19-36.

[21] S.N. Wood, Generalized Additive Models: an Introduction with R, Chapman and Hall/CRC, London, 2006.

[22] R.J Hyndman, S. Razbash, D. Schmidt, forecast: Forecasting functions for time series and linear models, R package version 3.19 (2012).

[23] T. Hanzák, Holt-Winters method with general seasonality, Kybernetika 48 (2012) 1-15.

[24] S. Boyd, L. Vandenberghe, Convex Optimization, Cambridge University Press, Cambridge, 2004.

[25] Redes Energéticas Nacionais (REN), Manual de procedimentos do gestor do sistema, (2008). (in Portuguese)

[26] K. Agnew, R. Burke, P. Ham-Su, Participation of demand response resources in ISO New England's ancillary service markets, Proc. of the Inter. Energy Prog. Eval. Conf. (2008).

[27] REN market data Jul. 2012. [Online] http://www.mercado.ren.pt/Paginas/default.aspx (accessed in November 2012)

[28] F.J. Soares, Impact of the deployment of electric vehicles in grid operation and expansion, PhD Thesis, Faculty of Engineering from the University of Porto (2011).

[29] M. Herrera, L. Torgo, J. Izquierdo, R. Pérez-García, Predictive models for forecasting hourly urban water demand, J. of Hydro. 387 (2010) 141-150.

[30] R.J. Bessa, M.A. Matos, Global against divided optimization for the participation of an EV aggregator in the day-ahead electricity market - part II: numerical analysis, Elect. Power Sys. Res. 95 (2013) 319-329.

[31] Ancillary Services, PJM State \& Member Training Department, 2013. [Online] http://www.pjm.com/ /media/training/core-curriculum/ip-gen-301/gen-301-ancillary-services.ashx (accessed in February 2013) 
Table 1: Set of charging solutions of an EV offering upward reserve power in a six-hour availability period with a charging requirement of $9 \mathrm{kWh}$.

(a)

\begin{tabular}{ccccccc}
\hline & $\mathrm{H} 1$ & $\mathrm{H} 2$ & $\mathrm{H} 3$ & $\mathrm{H} 4$ & $\mathrm{H} 5$ & $\mathrm{H} 6$ \\
\hline$E_{k}[\mathrm{kWh}]$ & 3 & 3 & 3 & 0 & 3 & 3 \\
$P_{k}^{u p}[\mathrm{~kW}]$ & 0 & 0 & 0 & 0 & 3 & 3 \\
\hline
\end{tabular}

(b)

\begin{tabular}{ccccccc}
\hline & $\mathrm{H} 1$ & $\mathrm{H} 2$ & $\mathrm{H} 3$ & $\mathrm{H} 4$ & $\mathrm{H} 5$ & $\mathrm{H} 6$ \\
\hline$E_{k}[\mathrm{kWh}]$ & 3 & 3 & 3 & 3 & 3 & 3 \\
$P_{k}^{u p}[\mathrm{~kW}]$ & 3 & 0 & 3 & 0 & 3 & 0 \\
\hline
\end{tabular}

Table 2: Example of a charging solution of an EV offering upward and downward reserve power in a six-hour availability period with a charging requirement of $9 \mathrm{kWh}$.

\begin{tabular}{ccccccc}
\hline & $\mathrm{H} 1$ & $\mathrm{H} 2$ & $\mathrm{H} 3$ & $\mathrm{H} 4$ & $\mathrm{H} 5$ & $\mathrm{H} 6$ \\
\hline$E_{k}[\mathrm{kWh}]$ & 2 & 2 & 2 & 3 & 2 & 2 \\
$P_{k}^{\text {up }}[\mathrm{kW}]$ & 0 & 1 & 1 & 0 & 2 & 0 \\
$P_{k}^{\text {down }}[\mathrm{kW}]$ & 0 & 0.5 & 0.5 & 0 & 1 & 0 \\
\hline
\end{tabular}

Table 3: MAPE and PBIAS of the aggregated availability and charging requirement forecast of fleets $A$ and $B$.

\begin{tabular}{ccccc}
\hline & \multicolumn{2}{c}{ Availability } & Charging Requirement \\
& MAPE & PBIAS & MAPE & PBIAS \\
\hline Fleet A & $6.99 \%$ & $4.45 \%$ & $29.93 \%$ & $5.75 \%$ \\
Fleet B & $8.09 \%$ & $-4.60 \%$ & $30.69 \%$ & $-5.86 \%$ \\
\hline
\end{tabular}


Table 4: MAE and RMSE of the forecasted energy and reserve prices (average values of 30 samples).

\begin{tabular}{ccccc}
\hline & \multicolumn{2}{c}{ Day-ahead } & \multicolumn{2}{c}{ Hour-ahead } \\
& MAE & RMSE & MAE & RMSE \\
\hline Elect. Energy Price [€/MWh] & 5.3 & 7.2 & - & - \\
Up. Res. Price [€/MWh] & 11.9 & 17.2 & 9.2 & 14.4 \\
Down. Res. Price [€/MWh] & 13.5 & 16.9 & 10.2 & 13.2 \\
Reserve Cap. Price [€/MW] & 4.4 & 5.7 & - & - \\
\hline
\end{tabular}




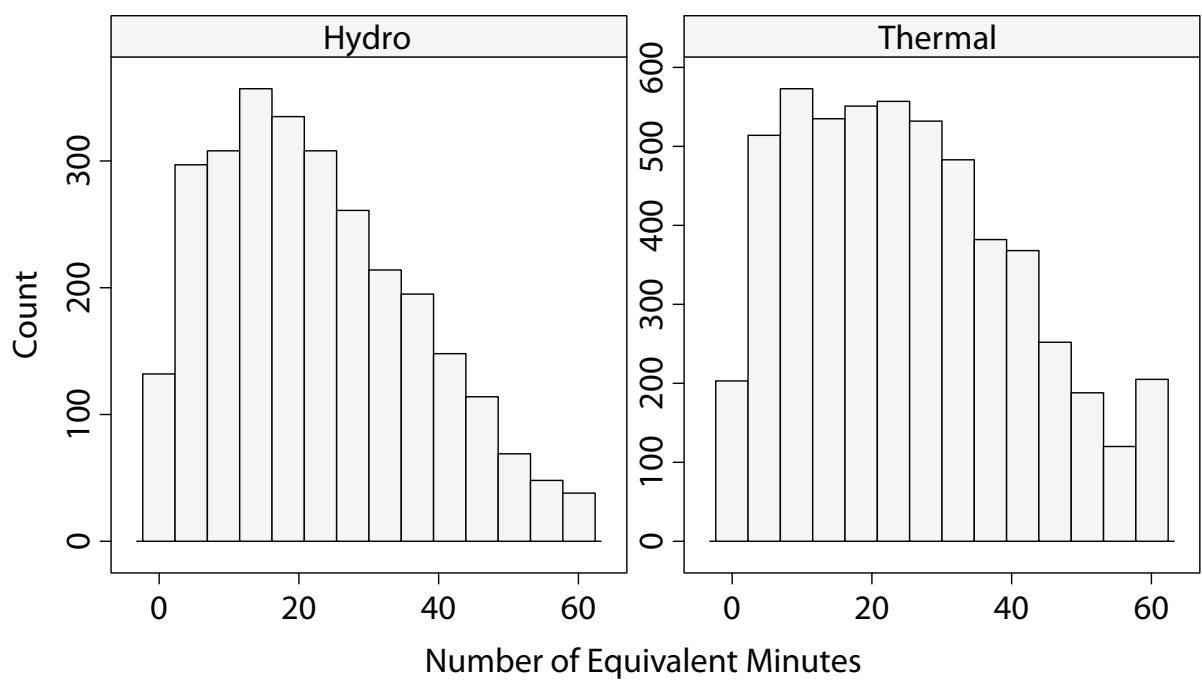

Figure 1: Histograms for the number of equivalent minutes of the upward secondary reserve of a hydro (Alqueva) and thermal (Lares) power plants in Portugal for the year 2011.

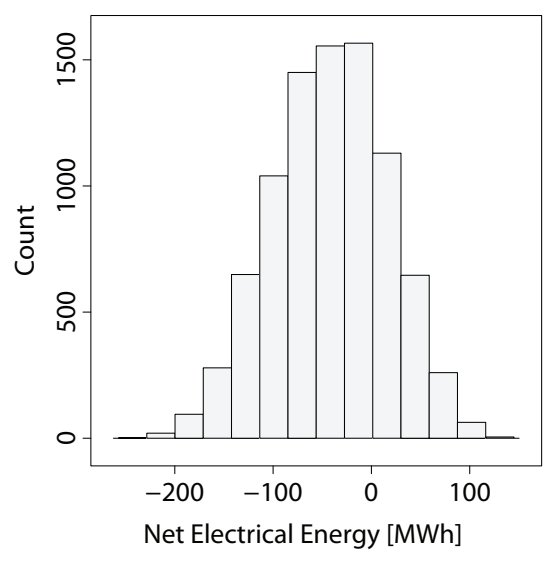

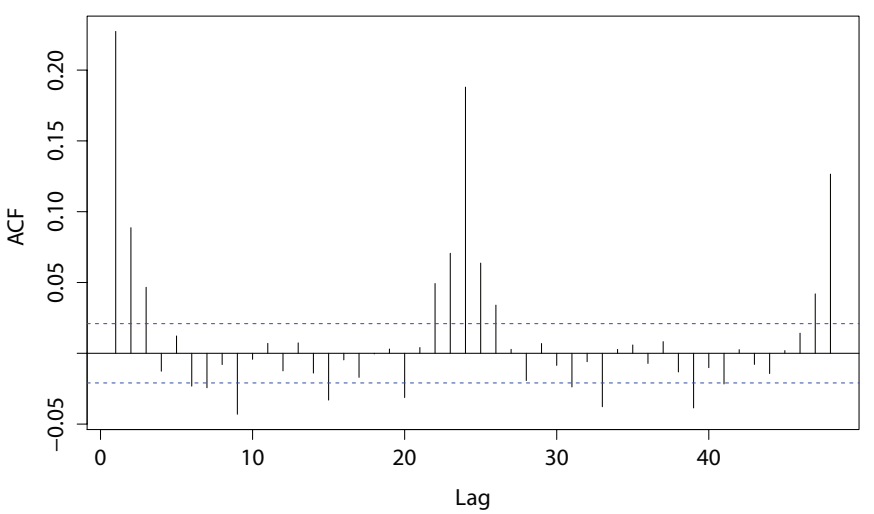

(b)

Figure 2: (a) Histogram of the net electrical energy of secondary reserve in Portugal for the year

2011 (negative value is upward reserve, positive is downward reserve); (b) Autocorrelation function (ACF) of the net electrical energy of secondary reserve in Portugal for the year 2011. 


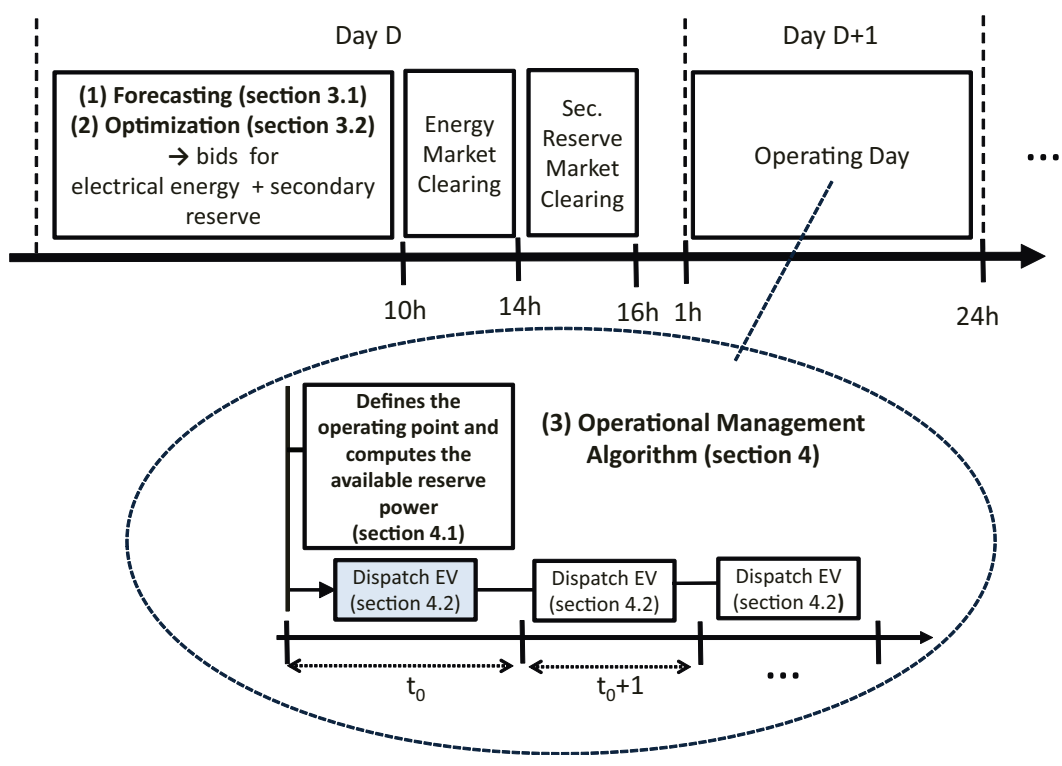

Figure 3: Sequence of tasks for the participation in the day-ahead energy and automatic reserve markets.

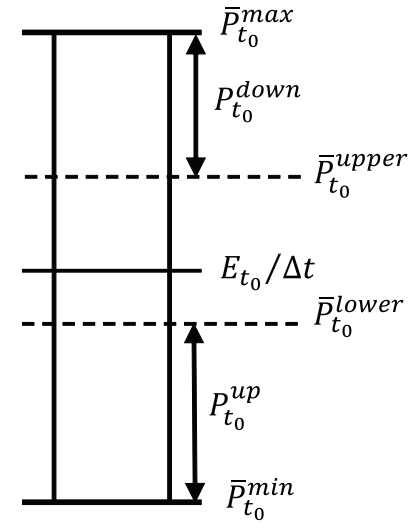

(a)

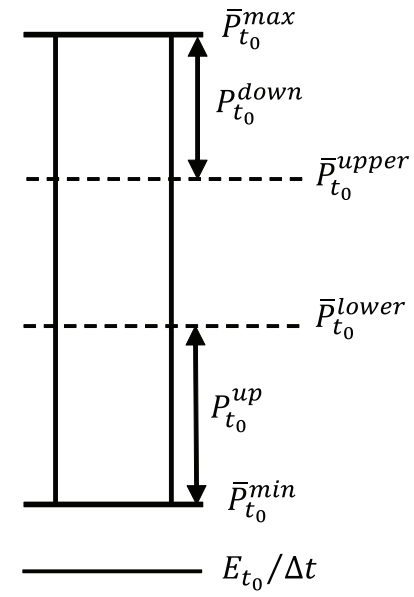

(b)

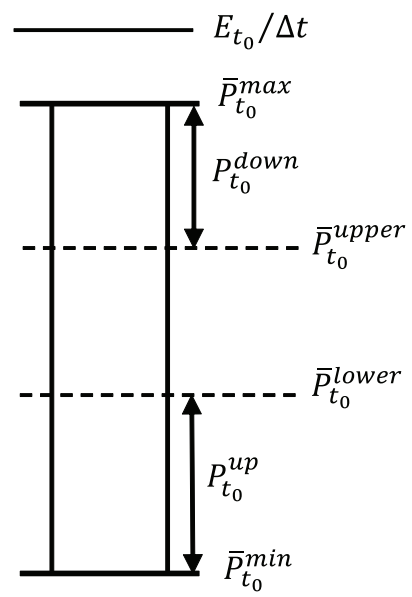

(c)

Figure 4: Variables required to redefine the operating point of the EV fleet. 


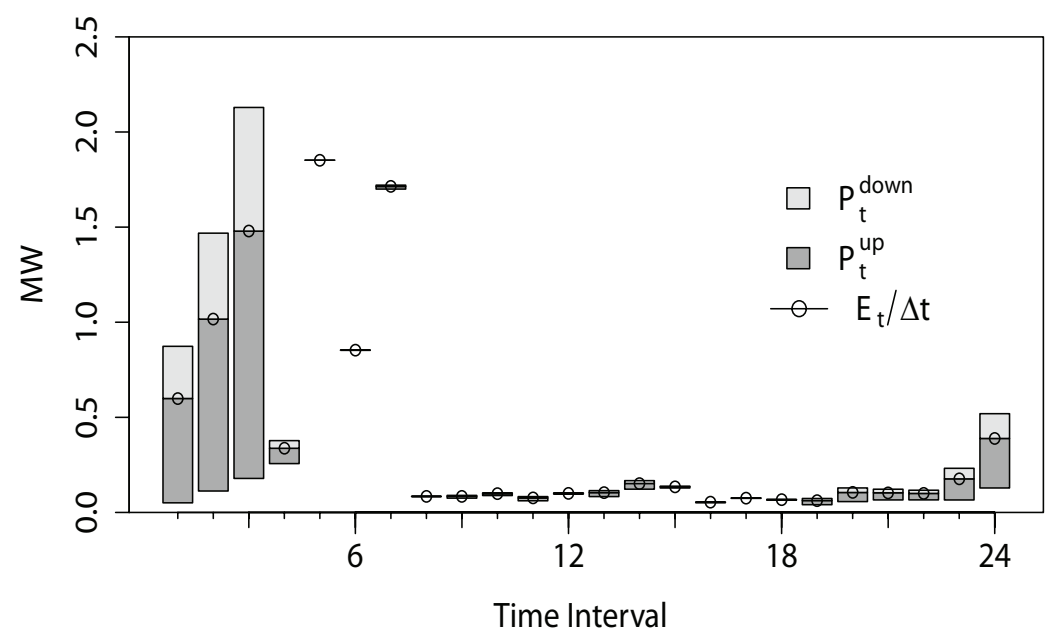

(a)

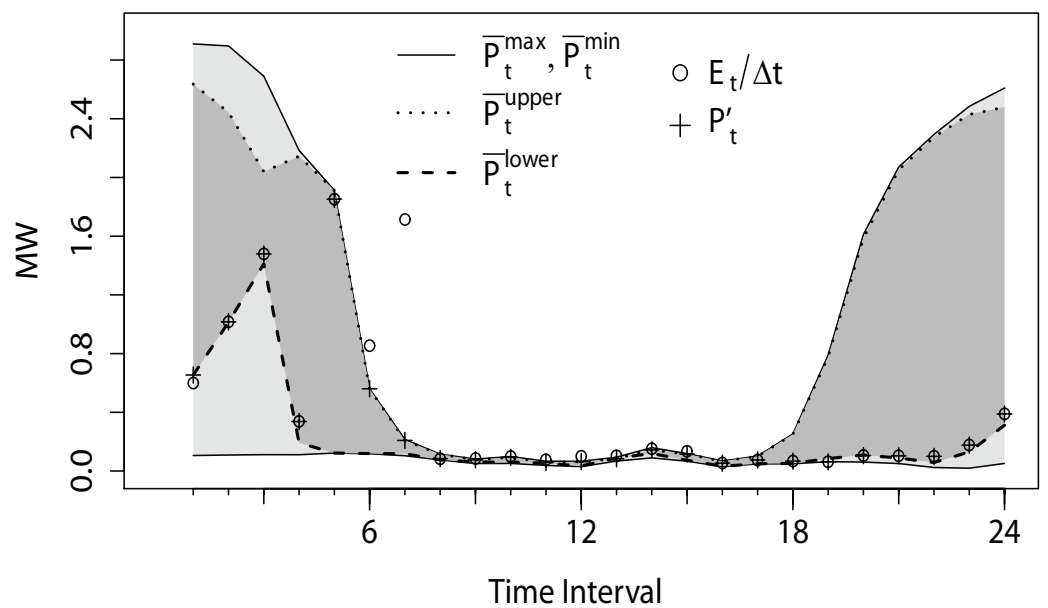

(b)

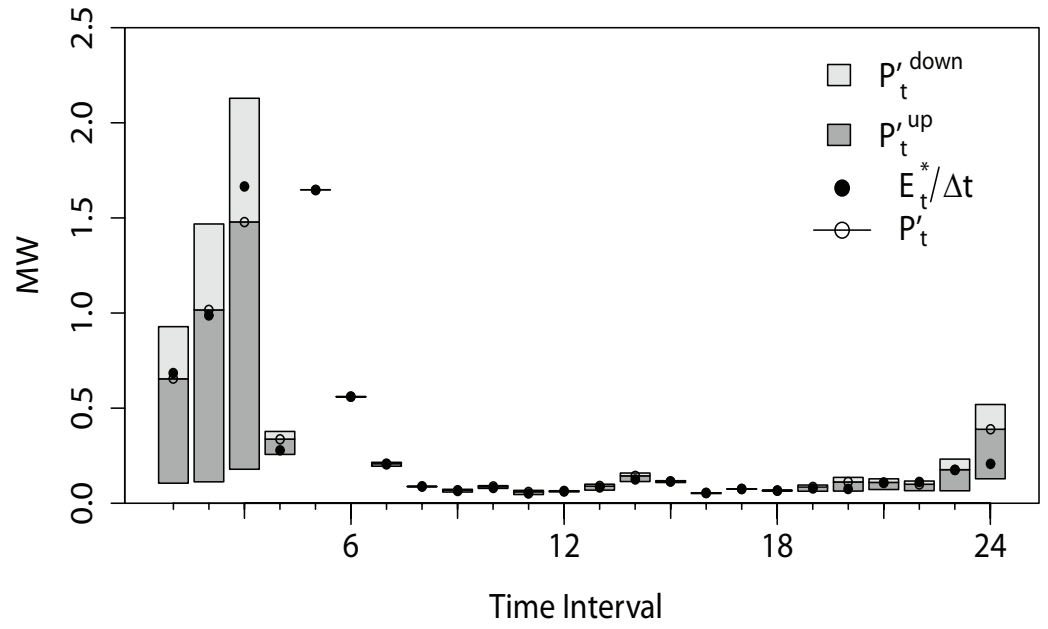

(c)

Figure 5: (a) Output of the day-ahead optimization (energy and secondary reserve power bids); (b) calculation of the redefined EV fleet operating point; (b) operating point, available upward and downward reserve power, and electrical energy consumed by the EV fleet during the operating interval. 


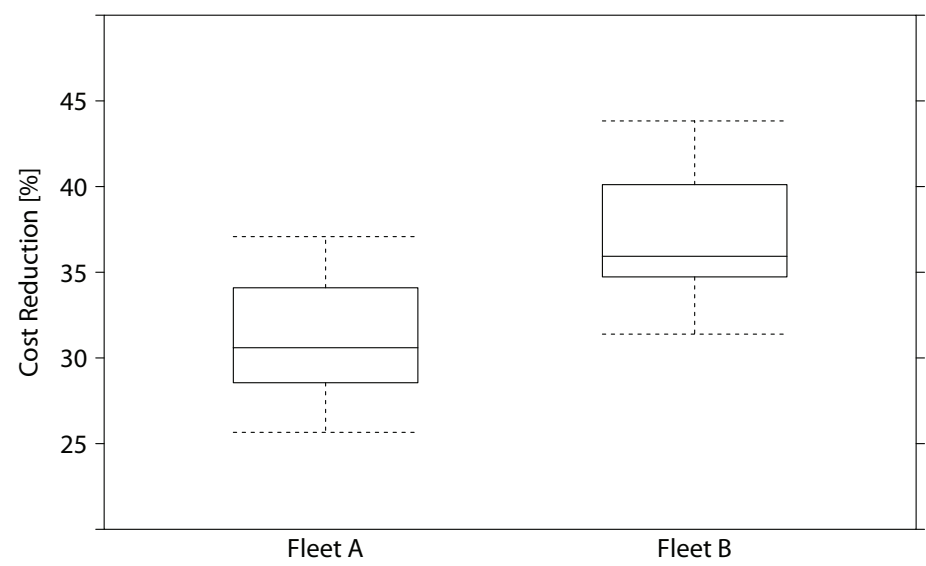

(a)

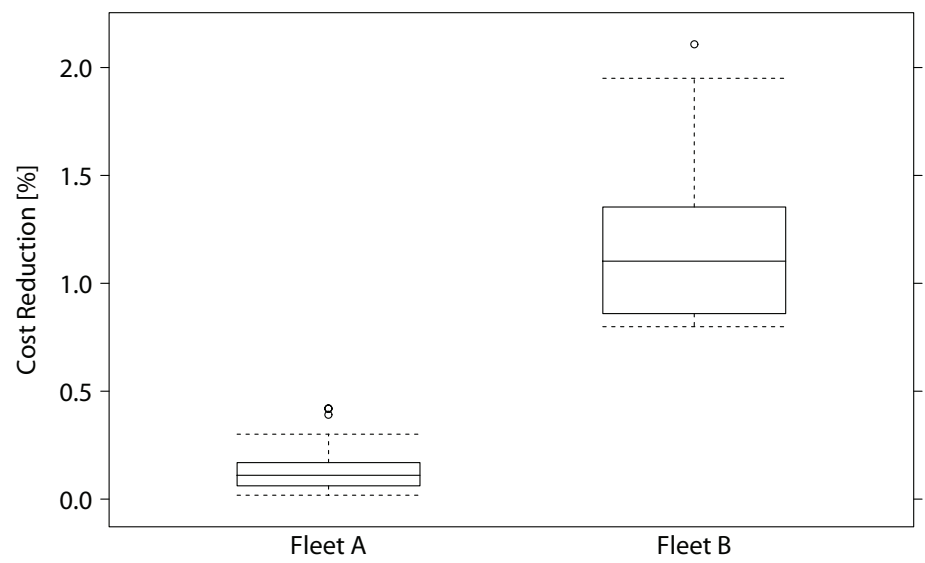

(b)

Figure 6: (a) Total cost reduction in fleets A and B for settlement scheme (1), using the participation in the energy market as reference; (b) total cost reduction of scheme (2) compared to scheme (1).

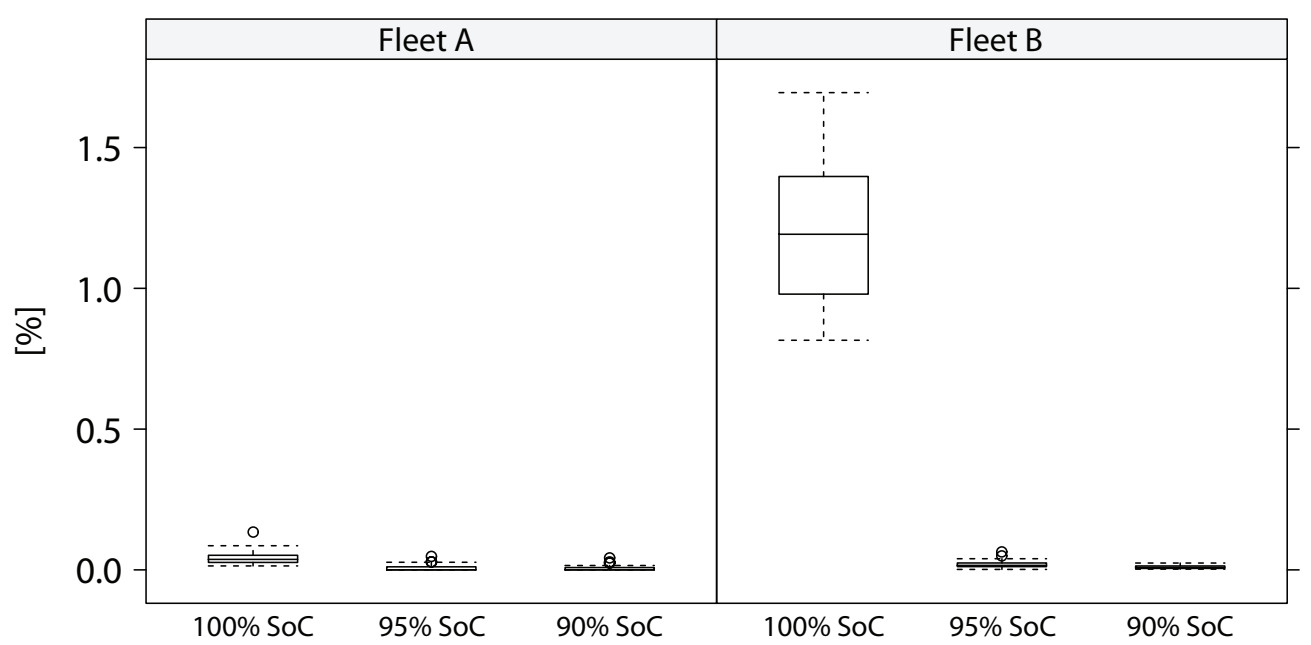

Figure 7: Percentage of reserve capacity shortage (pRCS) in fleets A and B. 


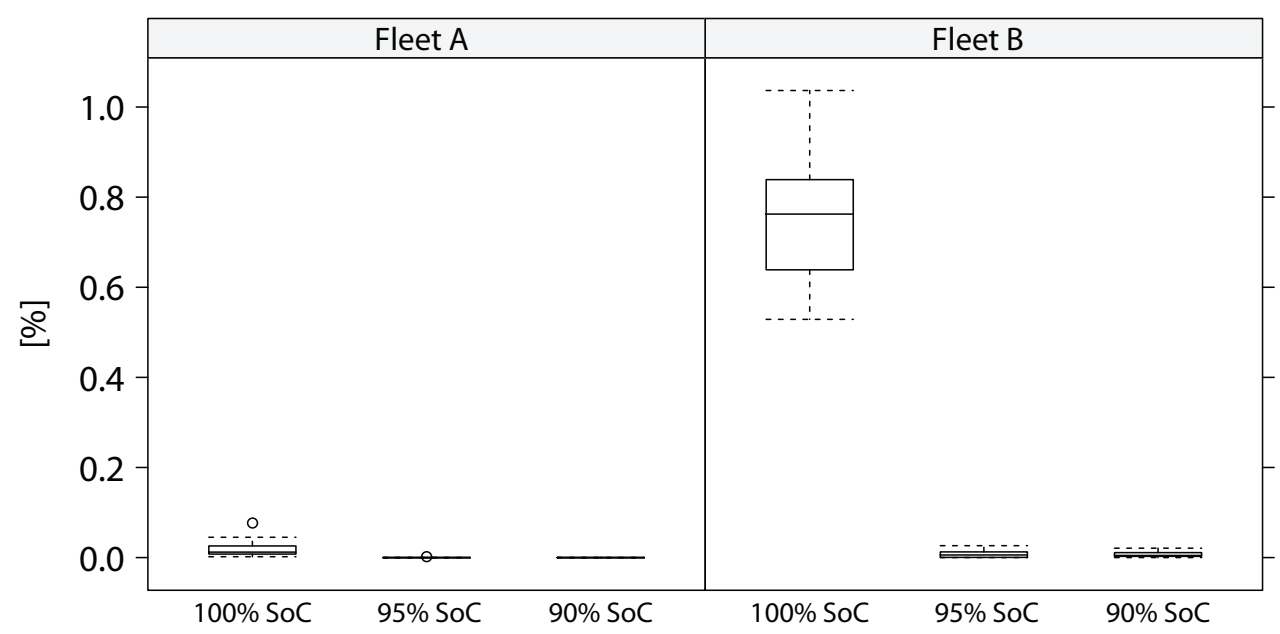

Figure 8: Percentage of upward reserve not supplied (pRNS) in fleets A and B.

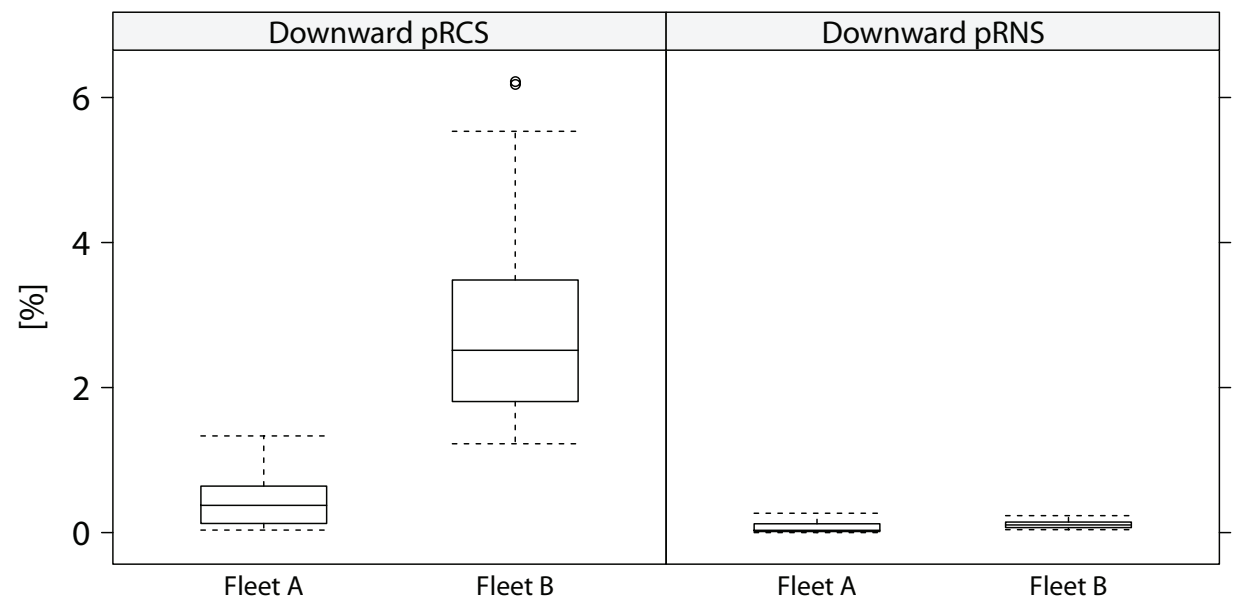

Figure 9: pRCS and pRNS of downward reserve in fleets $A$ and $B$.

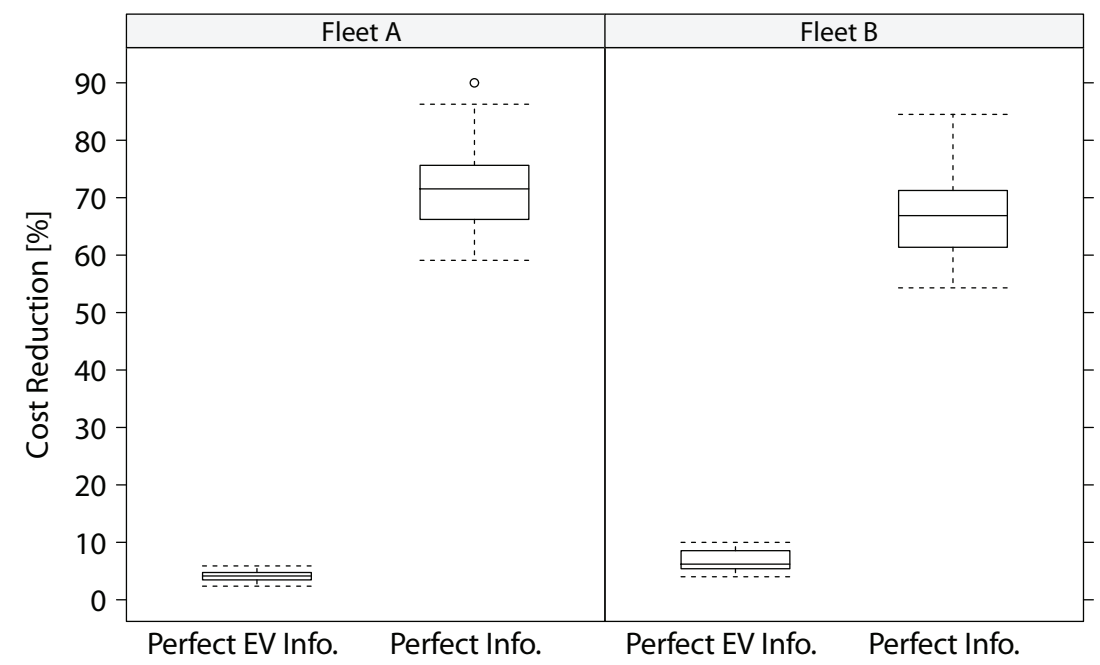

Figure 10: Reduction in the total cost for both fleets with two different sets of available information: perfect forecast for the EV variables; perfect forecast for all the variables. The reference is the result obtained with forecasts for all the variables. 